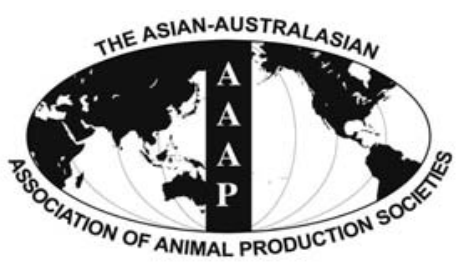

Asian-Aust. J. Anim. Sci.

Vol. 23, No. 1 : 131 - 148

January 2010

www.ajas.info

\title{
Spray Dried Animal Plasma as an Alternative to Antibiotics in Weanling Pigs* - A Review -
}

\author{
David Torrallardona** \\ IRTA, Ctra. Reus-El Morell, km. 3.8, E-43120 Constantí (Tarragona), Spain
}

\begin{abstract}
Piglet health at weaning is compromised due to several stress factors. Following the ban of antibiotic growth promoters new alternatives are required to control these problems. This paper reviews the evidence available for the use of spray dried animal plasma (SDAP) as an alternative to antibiotics in weaning pigs. Data from 75 trials in 43 publications involving over 12,000 piglets (mean values) have been used to calculate the performance responses of piglets according to several factors including SDAP origin, protein source from the control diet being replaced, dose of inclusion, age and weight of the piglets at weaning, sanitary conditions and simultaneous use or not of medication. Although the use of SDAP of all origins results in positive responses, it appears that plasma from porcine origin has the highest efficacy. This could be explained by the specificity of its IgG against porcine pathogens. During the first week post-weaning the response to plasma appears to increase with the inclusion dose, although over the two-week pre-starter period an optimal inclusion level of $4-8 \%$ is suggested. SDAP improves feed efficiency more markedly when the piglets are challenged with an experimental infection or when feed does not contain medication, which could be indicative of a lower expenditure of energy and nutrients to build an immune response against the challenge. There is evidence supporting that SDAP IgG and other bioactive substances therein prevent the binding of pathogens to the gut wall and reduce the incidence of diarrhoea in the post-weaning phase. Overall, plasma can be postulated as an excellent alternative to in-feed antimicrobials for piglets in the post-weaning phase. (Key Words : Spray Dried Animal Plasma, Pigs, Antibiotic Alternatives)
\end{abstract}

\section{INTRODUCTION}

Weaning is the most critical stage in pig production. Pigs at weaning are suddenly removed from the sow into a new environment where they have to change from suckling to eating dry feed from a hopper and drinking water from a drinker. Additionally at the same time they are mixed with pigs from other litters and fighting to establish new hierarchies occurs. As a result piglets go through a period of anorexia that compromises the functionality and integrity of the intestinal mucosa (van Beers-Schreurs et al., 1998). Once this period of starvation is over, the hungry piglet eats more feed than its gastrointestinal tract can cope with. The mucosa has lost its integrity and has not yet adapted to producing enzymes for digesting feed of vegetable origin (Miller et al., 1986). All this may increase the amount of

\footnotetext{
* Support from the European Animal Protein Association (EAPA), Brussels, Belgium is gratefully acknowledged.

** Corresponding Author: David Torrallardona. Tel: +34-977-3284-24, Fax: +34-977-34-40-55, E-mail: david.torrallardona@irta.es Received October 30, 2007; Accepted March 16, 2009
}

undigested feed in the gut and increase the animal's susceptibility to pathological disorders. These problems have been traditionally controlled with the use of antibiotics at subtherapeutical doses as growth promoters. However the use of antimicrobial growth promoters has been banned in the European Union due to concerns about the development of antimicrobial resistance that could be transferred to human pathogenic bacteria. However as observed the Danish antibiotic use monitoring programme (DANMAP, 2004), since their ban antimicrobial growth promoters have increasingly been replaced by prescribed therapeutic antibiotics. Veterinary prescription of antibiotics in Denmark has increased continously from 48 tonnes in 1996 to 112.5 tonnes in 2004. In the case of pigs in particular, it increased by $10 \%$ between 2003 and 2004. Thus several products, including spray dried animal plasma (SDAP), have been postulated as alternatives. The effect of SDAP on piglet performance has been previously reviewed (Coffey and Cromwell, 2001; van Dijk et al., 2001a). The objective of this paper is to review the evidence available to support the use of spray dried animal plasma as an alternative to antibiotics in weaning pigs. 


\section{WEANING}

At weaning, the supply of the beneficial factors provided by sow's milk to the piglet is suddenly stopped. Among these epidermal growth factor, polyamines, insulin and insulin like growth factor have been reported to contribute to the development of the intestinal tract (Pluske et al., 1997). Sow's milk also contains protective antibodies, the main immunoglobulin in milk being IgA (Husband and Bennell, 1980).

After the first days of age, the IgA and other milk immunoglobulins are not absorbed at the intestinal level; they provide continuous defence throughout the intestinal lumen against infectious organisms to which the sow is resistant (Svendsen and Larsen, 1977). This protection is essential while the piglet's own IgA production has not completely developed; this does not occur until 6-8 weeks of age (Svendsen and Brown, 1973). Therefore piglets weaned at 3-4 weeks of age lose the protection from maternal antibodies and cannot efficiently fight infections. It has been estimated that by 3 to 4 weeks of age the piglet receives approximately $1.6 \mathrm{~g}$ of $\mathrm{IgA} / \mathrm{d}$ (Svendsen and Brown, 1973). Ideally the piglet should be offered sow's milk for the first days post-weaning. Unfortunately this is not possible, but an attempt to use ingredients with similar characteristics is feasible. The sow's mammary epithelium transforms precursors from blood or interstitial fluid into milk constituents by different processes. Among these transcytosis is of special interest; by this mechanism, intact proteins, immumoglobulins, hormones and growth factors can cross the mammary epithelium and be secreted intact in the milk (Hunziker and Kraehenbuhl, 1998; Monks and Neville, 2004). Many of the components in sow's milk may also be present in SDPP, and therefore SDPP may be a good substitute for sow's milk.

At weaning, piglets are producing enzymes that are adapted to the digestion of the nutrients contained in sow's milk. Appreciable quantities of lipase, amylase and other enzymes required to digest the nutrients in dry feed are not produced until 3-4 weeks of age and an adequate production is not achieved until 8 weeks of age (Kidder and Manners, 1980; Jensen et al., 1997). In addition, the piglet's ability to produce hydrochloric acid in the stomach at weaning is limited (Easter, 1988). During lactation, the $\mathrm{pH}$ of the stomach is kept low due to conversion of lactose to lactic acid by the lactic acid bacteria (Lactobacillus and Bifidobacteria). At weaning, the lactose supply is suddently reduced and a feed with high buffer capacity is provided, which results in an increased $\mathrm{pH}$ of stomach digestive contents. The increased $\mathrm{pH}$ causes the loss of the acidic protective barrier against the entry of germs into the small intestine, and an inefficient protein digestion may occur
(Easter, 1988). Furthermore, at weaning the piglet has to learn to eat a new dry feed and to drink water. As a result piglets take on average about $15 \mathrm{~h}$ to initiate feed intake, but in some cases they may take up to 48 hours (Bruininx et al., 2001). The use of highly palatable ingredients may reduce the time to initiation to eating and ameliorate many of the problems associated with post-weaning anorexia. The poor feed intake associated with weaning results in a reduction of the height of the intestinal villi to almost half of their height before weaning. The mitotic activity in the crypts increases to compensate for this loss of enterocytes, their depth increases and the maturity of the enterocytes is reduced (Hampson, 1986; Miller et al., 1986; Kelly et al., 1991; Pluske et al., 1991). As well as the reduction in the small intestinal absorption area, the high proportion of immature enterocytes also compromises the enzymatic activity at the tips of the villi (Miller et al., 1986). The longer the piglet takes to iniciate eating, the hungrier it is and so eats in excess. As enzyme production is not appropiate due to the previous low feed intake (Makkink et al., 1994) feed cannot be properly digested and it may give undesirable microflora the chance to proliferate and cause GIT disorders.

\section{SPRAY DRIED ANIMAL PLASMA AS A PROTEIN SOURCE}

Animal plasma is a by-product from the abattoir, obtained from animal blood. The cellular fraction of blood is separated by centrifugation with the use of an anticoagulant. Plasma is then concentrated by vacuum evaporation or by filtration with inverse osmotic membranes or ultrafiltration and is finally dried by the spray technique, thus obtaining the so-called "spray dried animal plasma” (SDAP). During spray drying, plasma proteins are exposed to high temperatures for a very short period of time, and this has the advantage over conventional drying that the proteins are not denaturalised and preserve their biological activity (Gatnau et al., 1989; Borg et al., 2002). According to its origin we can classify SDAP into "spray dried porcine plasma" (SDPP) and "spray dried bovine plasma” (SDBP), which are of porcine and bovine origin, respectively. It has been suggested that the beneficial effects of spray dried plasma are related to its immunoglobulin content (Gatnau and Zimmerman, 1991), and for this reason, some SDAP sources have an standardised IgG content. In addition, sources of plasma that are enriched for some specific immunoglobulins, have also been obtained from pigs that have been vaccinated against specific pathogens for this purpose. The plasma obtained with this procedure is known as spray dried immune porcine plasma (SDIPP). Plasma sources can also differ according to the use of technological processes (e.g. 
Table 1. Essential amino acid composition and apparent ileal digestibility of different spray dried plasma sources

\begin{tabular}{|c|c|c|c|c|c|}
\hline & \multicolumn{3}{|c|}{ AA content (\%) } & \multicolumn{2}{|c|}{ SDPP Ileal digestibility for piglets (\%) } \\
\hline & SDAP $^{1}$ & $\mathrm{SDPP}^{2}$ & $\mathrm{SDBP}^{2}$ & Apparent & True \\
\hline Arginine & 4.55 & 4.47 & 4.70 & 73 & 87 \\
\hline Histidine & 2.55 & 2.51 & 2.45 & 77 & 88 \\
\hline Isoleucine & 2.71 & 2.79 & 2.53 & 77 & 88 \\
\hline Leucine & 7.61 & 7.44 & 7.63 & 77 & 87 \\
\hline Lysine & 6.84 & 6.84 & 7.43 & 76 & 88 \\
\hline Methionine & 0.75 & 0.62 & 0.95 & 72 & 82 \\
\hline Cystine & 2.63 & 3.03 & 3.16 & - & - \\
\hline Phenylalanine & 4.42 & 4.43 & 4.25 & 75 & 86 \\
\hline Tyrosine & 3.53 & 3.79 & 3.89 & - & - \\
\hline Threonine & 4.72 & 4.54 & 5.54 & 71 & 84 \\
\hline Tryptophan & 1.36 & 1.36 & 1.45 & - & - \\
\hline Valine & 4.94 & 5.07 & 5.64 & 72 & 84 \\
\hline
\end{tabular}

${ }^{1}$ NRC (1988). ${ }^{2}$ van der Peet-Schwering and Binnnendijk (1997). ${ }^{3}$ Yun et al. (2005).

ultrafiltration) to reduce the salt content before spray drying. Studies comparing plasma with high and low ash content have not found differences (Gatnau and Zimmerman, 1994; Rantanen et al., 1994; Russell, 1994). However these plasma sources have been tested at inclusion levels between $2-7.5 \%$. It is possible that the high ash content could be detrimental at higher plasma inclusion levels.

A hygienic collection and processing of the blood is essential to ensure a good quality of plasma. Irradiation or antibacterial chemical treatment with formaldehyde of plasma has been shown to improve the efficacy of feed grade plasma (DeRouchey et al., 2004). However the same authors could not find an effect in plasma of low bacterial contamination (food grade). The safety of plasma as possible vector for transmissible diseases of pigs must be also taken into consideration (van Dijk et al., 2001a). Polo et al. (2005) have demonstrated that the spray drying process is effective in inactivating pseudorabies and porcine

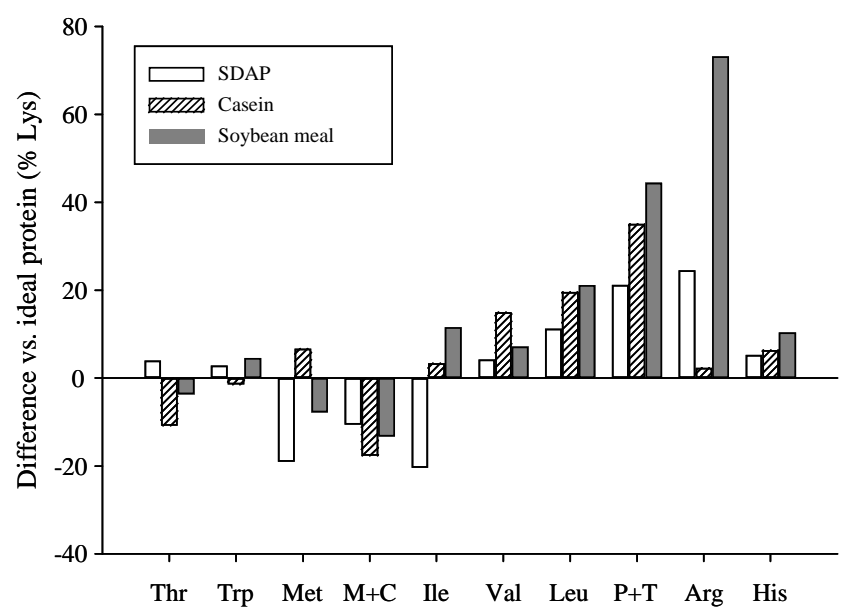

Figure 1. Deviation of the amino acid profiles of spray dried plasma, casein and soybean meal from the piglet's ideal protein profile (calculated after NRC, 1998). respiratory and reproductive syndrome viruses. The same authors have also shown that functional antibodies against porcine parvovirus in SDAP fed to naïve pigs did not promote their seroconversion.

Plasma is composed of protein, minerals and water. Proteins constitute the most important fraction; 95\% of these are albumins and globulins (Tumbleson et al., 1986). The albumins are proteins whose function is the maintenance of plasma osmotic pressure and provision of the buffering capacity of the blood. Within the globulins, the gamma globulins have an immune function, of which IgG are the most important functional fraction of blood plasma. The other types, IgM, IgA, IgD and IgE are minor in blood plasma. The protein content in plasma ranges between 70 and $80 \%$ depending on the technological processes applied during its production. High protein levels are usually related to low ash contents and vice versa. Plasma sources concentrated by ultrafiltration have a higher protein concentration than those concentrated by reverse osmosis or vacuum drying. The amino acid composition of different spray dried plasma sources and their amino acid ileal digestibilities for weaning piglets are reported in Table 1. There are no substantial differences in amino acid composition between the plasma from porcine or bovine origin. Relative to the requirements of weaning pigs, the amino acid composition of plasma protein is well balanced, except for methionine and isoleucine which are limiting (Figure 1; NRC, 1998). The nutritive value of SDAP is similar to that of other high quality proteins, and cannot explain on its own the positive effects observed on the performance of weaning pigs. For example Kim and Easter (2001) did not find differences in lysine availability between SDAP and fishmeal, and Chae et al. (1999) observed that SDAP had a lower ileal protein digestibility than dried skim milk, isolated soy protein, and wheat gluten. 
EFFECTS OF SDAP ON THE GROWTH PERFORMANCE OF NURSERY PIGS

A large amount of work on the efficacy of SDAP has been published since it was first proposed as a protein source for piglets in the late eighties (Zimmerman, 1987; Gatnau and Zimmerman, 1990). Table 2 summarises the results of 75 trials in 43 publications involving over 12,000

Table 2. Summary of trials testing spray dried plasma as a protein source for piglets at weaning

\begin{tabular}{|c|c|c|c|c|c|c|c|c|c|c|c|c|c|c|c|c|c|}
\hline \multirow{2}{*}{ Ref. } & \multirow{2}{*}{ Plasma } & \multirow{2}{*}{ Dose } & \multirow{2}{*}{ Ref. Protein } & Alternative & Modication & Cbollongr & Piøc/tr & $\Delta \alpha$ & Whiøh & & First & t week & & & Pre-sta & irter pha & \\
\hline & & & & Product & Niecuicanon & Cnanenge & $\mathrm{PigS/LT}$ & Age & vveigi & $\mathrm{d}$ & ADG & ADFI & $\overline{\text { FGR }}$ & $\mathrm{d}$ & ADG & ADFI & FGR \\
\hline Torrallardona et al. (2007) & & - & FM & - & - & yes & 12 & 21 & 6.2 & - & - & - & - & 14 & 150 & 225 & 1.56 \\
\hline & SDAP & 6 & FM & - & - & yes & 12 & 21 & 6.2 & - & - & - & - & 14 & 223 & 301 & 1.35 \\
\hline & & - & FM & Ca formate & - & yes & 12 & 21 & 6.2 & - & - & - & - & 14 & 141 & 202 & 1.47 \\
\hline & & - & FM & Colistin & yes & yes & 12 & 21 & 6.2 & - & - & - & - & 14 & 173 & 236 & 1.37 \\
\hline Torrallardona et al. (2007) & & - & FM & - & - & yes & 11 & 21 & 7.4 & - & - & - & - & 10 & 112 & 192 & 1.78 \\
\hline & SDAP & 6 & FM & - & - & yes & 11 & 21 & 7.4 & - & - & - & - & 10 & 142 & 230 & 2.01 \\
\hline & & - & FM & Ca formate & - & yes & 11 & 21 & 7.4 & - & - & - & - & 10 & 106 & 172 & 1.65 \\
\hline & & - & FM & Colistin & - & yes & 11 & 21 & 7.4 & - & - & - & - & 10 & 128 & 196 & 1.52 \\
\hline Nofrarias et al. (2006) & & - & $\mathrm{SPC}+\mathrm{WG}$ & - & no & - & 8 & 20 & 6.0 & 7 & 40 & 139 & 3.33 & 14 & 125 & 239 & 1.89 \\
\hline & SDPP & 6 & $\mathrm{SPC}+\mathrm{WG}$ & - & no & - & 8 & 20 & 6.0 & 7 & 75 & 141 & 1.96 & 14 & 150 & 241 & 1.59 \\
\hline & & - & $\mathrm{SPC}+\mathrm{WG}$ & Plant extract & no & - & 8 & 20 & 6.0 & 7 & 74 & 192 & 2.78 & 14 & 166 & 301 & 1.82 \\
\hline Conde (2005) & & - & FM & - & - & yes & 15 & 21 & 7.3 & 7 & 123 & 181 & 1.47 & 14 & 182 & 263 & 1.47 \\
\hline & SDAP & 6 & FM & - & - & yes & 15 & 21 & 7.3 & 7 & 175 & 217 & 1.26 & 14 & 245 & 312 & 1.28 \\
\hline & SDAP & 6 & FM & - & - & yes & 15 & 21 & 7.3 & 7 & 133 & 186 & 1.44 & 14 & 199 & 275 & 1.37 \\
\hline & & - & FM & Colistin & - & yes & 15 & 21 & 7.3 & 7 & 176 & 207 & 1.23 & 14 & 252 & 312 & 1.26 \\
\hline Conde (2005) & & - & FM & - & - & yes & 12 & 21 & 5.7 & 7 & -25 & 134 & . & 14 & 7 & 171 & - \\
\hline & SDIPP & 6 & FM & - & - & yes & 12 & 21 & 5.7 & 7 & -9 & 104 & . & 14 & 72 & 172 & - \\
\hline & SDPP & 6 & FM & - & - & yes & 12 & 21 & 5.7 & 7 & 15 & 145 & . & 14 & 56 & 188 & - \\
\hline Pierce et al. (2005) & & - & SPC & - & - & - & 19 & 15 & 5.3 & 7 & 117 & 178 & 1.57 & 14 & 170 & 243 & 1.44 \\
\hline & SDPP & 8 & SPC & - & - & - & 19 & 15 & 5.3 & 7 & 102 & 187 & 1.99 & 14 & 228 & 356 & 1.57 \\
\hline & & - & SPC & IgG 64\% & - & - & 19 & 15 & 5.3 & 7 & 146 & 196 & 1.45 & 14 & 228 & 325 & 1.45 \\
\hline & & - & SPC & IgG $128 \%$ & - & - & 19 & 15 & 5.3 & 7 & 180 & 225 & 1.29 & 14 & 264 & 373 & 1.41 \\
\hline & & - & SPC & IgG $192 \%$ & - & - & 19 & 15 & 5.3 & 7 & 167 & 204 & 1.24 & 14 & 258 & 356 & 1.39 \\
\hline Pierce et al. (2005) & & - & SPC & - & - & - & 19 & 20 & 5.6 & 7 & 99 & 209 & 2.14 & 14 & 174 & 285 & 1.65 \\
\hline & SDPP & 8 & SPC & - & - & - & 19 & 20 & 5.6 & 7 & 206 & 352 & 1.73 & 14 & 267 & 451 & 1.69 \\
\hline & SDBP & 8 & SPC & - & - & - & 19 & 20 & 5.6 & 7 & 141 & 264 & 1.93 & 14 & 194 & 336 & 1.76 \\
\hline & & - & SPC & Bov IgG $50 \%$ & - & - & 19 & 20 & 5.6 & 7 & 182 & 270 & 1.52 & 14 & 223 & 352 & 1.60 \\
\hline & & - & SPC & Bov IgG 100\% & - & - & 19 & 20 & 5.6 & 7 & 202 & 296 & 1.49 & 14 & 254 & 391 & 1.54 \\
\hline Pierce et al. (2005) & & - & SPC & - & - & - & 19 & 23 & 6.4 & 7 & 87 & 175 & 2.25 & 14 & 228 & 333 & 1.47 \\
\hline & SDPP & 8 & SPC & - & - & - & 19 & 23 & 6.4 & 7 & 183 & 287 & 1.60 & 14 & 265 & 425 & 1.62 \\
\hline & SDBP & 8 & SPC & - & - & - & 19 & 23 & 6.4 & 7 & 157 & 256 & 1.75 & 14 & 268 & 404 & 1.53 \\
\hline & & - & SPC & Bov IgG $50 \%$ & - & - & 19 & 23 & 6.4 & 7 & 162 & 235 & 1.46 & 14 & 287 & 389 & 1.35 \\
\hline & & - & SPC & Bov IgG $100 \%$ & - & - & 19 & 23 & 6.4 & 7 & 150 & 229 & 1.66 & 14 & 271 & 380 & 1.43 \\
\hline Pierce et al. (2005) & & - & SPC & - & - & - & 16 & 21 & 5.6 & 7 & 141 & 311 & 2.35 & 14 & 252 & 438 & 1.74 \\
\hline & SDPP & 8 & SPC & - & - & - & 16 & 21 & 5.6 & 7 & 229 & 462 & 2.10 & 14 & 281 & 500 & 1.81 \\
\hline & & - & SPC & IgG & - & - & 16 & 21 & 5.6 & 7 & 241 & 410 & 1.99 & 14 & 262 & 443 & 1.74 \\
\hline & & - & SPC & Albumin & - & - & 16 & 21 & 5.6 & 7 & 150 & 297 & 2.16 & 14 & 228 & 359 & 1.74 \\
\hline & & - & SPC & LMW & - & - & 16 & 21 & 5.6 & 7 & 119 & 300 & 2.77 & 14 & 223 & 416 & 1.88 \\
\hline Pierce et al. (2005) & & - & SPC & - & - & - & 16 & 21 & 6.3 & 7 & 162 & 216 & 1.37 & 14 & 274 & 386 & 1.43 \\
\hline & SDPP & 8 & SPC & - & - & - & 16 & 21 & 6.3 & 7 & 272 & 376 & 1.42 & 14 & 334 & 461 & 1.40 \\
\hline & & - & SPC & $\operatorname{IgG} 40 \%$ & - & - & 16 & 21 & 6.3 & 7 & 228 & 291 & 1.27 & 14 & 334 & 461 & 1.40 \\
\hline & & - & SPC & IgG $80 \%$ & - & - & 16 & 21 & 6.3 & 7 & 272 & 347 & 1.30 & 14 & 333 & 471 & 1.42 \\
\hline & & - & SPC & IgG $120 \%$ & - & - & 16 & 21 & 6.3 & 7 & 273 & 317 & 1.18 & 14 & 349 & 470 & 1.38 \\
\hline Bikker et al. (2004) & & - & $\mathrm{FM}+\mathrm{PP}$ & - & no & - & 72 & 26 & 8.0 & 7 & 176 & 194 & 1.13 & 14 & 249 & 303 & 1.22 \\
\hline & & - & $\mathrm{FM}+\mathrm{PP}$ & Avilamycin & yes & - & 72 & 26 & 8.0 & 7 & 187 & 196 & 1.06 & 14 & 266 & 317 & 1.20 \\
\hline & SDAP & 4 & $\mathrm{FM}+\mathrm{PP}$ & - & no & - & 72 & 26 & 8.0 & 7 & 231 & 228 & 0.99 & 14 & 295 & 339 & 1.15 \\
\hline & SDAP & 4 & $\mathrm{FM}+\mathrm{PP}$ & Avilamycin & yes & - & 72 & 26 & 8.0 & 7 & 222 & 215 & 0.97 & 14 & 288 & 328 & 1.14 \\
\hline Bikker et al. (2004) & & - & $\mathrm{FM}+\mathrm{PP}$ & - & no & - & 110 & 26 & 8.3 & 7 & 121 & 152 & 1.27 & 14 & 182 & 230 & 1.27 \\
\hline & & - & $\mathrm{FM}+\mathrm{PP}$ & - & no & - & 110 & 26 & 8.3 & 7 & 124 & 155 & 1.32 & 14 & 171 & 230 & 1.37 \\
\hline & SDAP & 4 & $\mathrm{FM}+\mathrm{PP}$ & - & no & - & 110 & 26 & 8.3 & 7 & 149 & 167 & 1.14 & 14 & 186 & 231 & 1.25 \\
\hline & SDAP & 4 & $\mathrm{FM}+\mathrm{PP}$ & - & no & - & 110 & 26 & 8.3 & 7 & 141 & 160 & 1.18 & 14 & 191 & 238 & 1.26 \\
\hline Bosi et al. (2004) & & - & FM & - & no & yes & 10 & 21 & 4.9 & - & - & - & - & 15 & 128 & 175 & 1.81 \\
\hline & SDAP & 6 & FM & - & no & yes & 12 & 21 & 4.9 & - & - & - & - & 15 & 148 & 186 & 1.48 \\
\hline & & - & FM & Antibiotics & yes & yes & 12 & 21 & 4.9 & - & - & - & - & 15 & 164 & 197 & 1.30 \\
\hline & SDAP & 6 & FM & Antibiotics & yes & yes & 12 & 21 & 4.9 & - & - & - & - & 15 & 186 & 214 & 1.30 \\
\hline DeRouchey et al. (2004) & & - & SBM46 & - & - & - & 30 & 17 & 5.9 & 5 & 223 & 209 & 0.93 & 10 & 239 & 281 & 1.18 \\
\hline & $\mathrm{SDAP}_{1}$ & 5 & SBM46 & - & - & - & 30 & 17 & 5.9 & 5 & 268 & 215 & 0.79 & 10 & 295 & 328 & 1.11 \\
\hline & $\operatorname{irSDAP}_{1}$ & 5 & SBM46 & - & - & - & 30 & 17 & 5.9 & 5 & 321 & 284 & 0.87 & 10 & 330 & 364 & 1.10 \\
\hline & $\mathrm{SDAP}_{2}$ & 5 & SBM46 & - & - & - & 30 & 17 & 5.9 & 5 & 223 & 212 & 0.96 & 10 & 253 & 293 & 1.16 \\
\hline & $\operatorname{irSDAP}_{2}$ & 5 & SBM46 & - & - & - & 30 & 17 & 5.9 & 5 & 280 & 238 & 0.84 & 10 & 297 & 325 & 1.09 \\
\hline & FDAP & 5 & SBM46 & - & - & - & 30 & 17 & 5.9 & 5 & 273 & 220 & 0.80 & 10 & 288 & 307 & 1.06 \\
\hline DeRouchey et al. (2004) & & - & SBM46 & - & - & - & 40 & 17 & 6.3 & 7 & 191 & 169 & 0.91 & 14 & 258 & 255 & 0.99 \\
\hline & $\mathrm{SDAP}_{1}$ & 5 & SBM46 & - & - & - & 40 & 17 & 6.3 & 7 & 233 & 205 & 0.88 & 14 & 281 & 279 & 0.99 \\
\hline & $\operatorname{irSDAP}_{1}$ & 5 & SBM46 & - & - & - & 40 & 17 & 6.3 & 7 & 253 & 215 & 0.85 & 14 & 292 & 285 & 0.97 \\
\hline & $\mathrm{SDAP}_{2}$ & 5 & SBM46 & - & - & - & 40 & 17 & 6.3 & 7 & 242 & 203 & 0.84 & 14 & 290 & 284 & 0.98 \\
\hline & $\operatorname{irSDAP}_{2}$ & 5 & SBM46 & - & - & - & 40 & 17 & 6.3 & 7 & 239 & 202 & 0.84 & 14 & 280 & 277 & 0.99 \\
\hline & $\mathrm{SDAP}_{3}$ & 5 & SBM46 & - & - & - & 40 & 17 & 6.3 & 7 & 255 & 219 & 0.85 & 14 & 299 & 293 & 0.98 \\
\hline & $\operatorname{irSDAP}_{3}$ & 5 & SBM46 & - & - & - & 40 & 17 & 6.3 & 7 & 262 & 226 & 0.86 & 14 & 288 & 287 & 0.99 \\
\hline & $\mathrm{SDAP}_{4}$ & 5 & SBM46 & - & - & - & 40 & 17 & 6.3 & 7 & 273 & 233 & 0.85 & 14 & 303 & 302 & 1.00 \\
\hline & $\operatorname{irSDAP}_{4}$ & 5 & SBM46 & - & - & - & 40 & 17 & 6.3 & 7 & 263 & 216 & 0.83 & 14 & 288 & 281 & 0.98 \\
\hline
\end{tabular}


Table 2. Summary of trials testing spray dried plasma as a protein source for piglets at weaning (Continued)

\begin{tabular}{|c|c|c|c|c|c|c|c|c|c|c|c|c|c|c|c|c|c|}
\hline \multirow{2}{*}{ Ref. } & \multirow{2}{*}{ Plasma } & \multirow{2}{*}{ Dose } & \multirow{2}{*}{ Ref. protein } & \multirow{2}{*}{$\begin{array}{l}\text { Alternative } \\
\text { product }\end{array}$} & Mdiction & Chollonas & Dir & $A_{\mathrm{s}}$ & Whicht & & Firs & t week & & & $\overline{\text { Pre-stal }}$ & rter pha & \\
\hline & & & & & Medication & Challenge & $\mathrm{Plgs} / \mathrm{tr}$ & Age & Welgnt & $\bar{d}$ & ADG & ADFI & $\overline{\text { FGR }}$ & $\mathrm{d}$ & ADG & ADFI & FGR \\
\hline DeRouchey et al. (2004) & & - & SBM46 & - & - & - & 35 & 17 & 6.1 & 7 & 102 & 105 & 1.02 & 14 & 226 & 235 & 1.04 \\
\hline & $\mathrm{SDAP}_{1}$ & 5 & SBM46 & - & - & - & 35 & 17 & 6.1 & 7 & 144 & 116 & 0.80 & 14 & 236 & 230 & 0.97 \\
\hline & $\operatorname{irSDAP}_{1}$ & 5 & SBM46 & - & - & - & 35 & 17 & 6.1 & 7 & 169 & 138 & 0.82 & 14 & 269 & 259 & 0.96 \\
\hline & $\mathrm{SDAP}_{2}$ & 5 & SBM46 & - & - & - & 35 & 17 & 6.1 & 7 & 183 & 158 & 0.85 & 14 & 261 & 256 & 0.98 \\
\hline & $\operatorname{irSDAP}_{2}$ & 5 & SBM46 & - & - & - & 35 & 17 & 6.1 & 7 & 195 & 161 & 0.83 & 14 & 270 & 265 & 0.98 \\
\hline Lawrence et al. (2004) & & - & SBM46 & - & yes & - & 42 & 21 & 6.2 & - & - & - & - & 14 & 312 & 319 & 1.14 \\
\hline & & - & WG & - & yes & - & 42 & 21 & 6.2 & - & - & - & - & 14 & 330 & 341 & 0.99 \\
\hline & SDAP & 1.25 & WG & - & yes & - & 42 & 21 & 6.2 & - & - & - & - & 14 & 318 & 322 & 0.97 \\
\hline & SDAP & 2.5 & WG & - & yes & - & 42 & 21 & 6.2 & - & - & - & - & 14 & 305 & 313 & 0.98 \\
\hline & SDAP & 3.75 & WG & - & yes & - & 42 & 21 & 6.2 & - & - & - & - & 14 & 319 & 327 & 0.95 \\
\hline & SDAP & 5 & WG \& SBM46 & - & yes & - & 42 & 21 & 6.2 & - & - & - & - & 14 & 294 & 301 & 0.95 \\
\hline Lawrence et al. (2004) & & - & SBM46 & - & yes & - & 40 & 21 & 7.0 & - & - & - & - & 14 & 319 & 325 & 0.93 \\
\hline & & - & SBM46+WG ${ }_{1}$ & - & yes & - & 40 & 21 & 7.0 & - & - & - & - & 14 & 320 & 315 & 0.88 \\
\hline & & - & $\mathrm{SBM} 46+\mathrm{WG}_{2}$ & - & yes & - & 40 & 21 & 7.0 & - & - & - & - & 14 & 303 & 306 & 0.93 \\
\hline & & - & WG & - & yes & - & 40 & 21 & 7.0 & - & - & - & - & 14 & 302 & 291 & 0.89 \\
\hline & SDAP & 5 & SBM46 \& WG & - & yes & - & 40 & 21 & 7.0 & - & - & - & - & 14 & 331 & 333 & 0.92 \\
\hline Lawrence et al. (2004) & & - & SBM46 & - & yes & - & 40 & 21 & 6.0 & - & - & - & - & 14 & 259 & 271 & 1.00 \\
\hline & & - & $\mathrm{hWG}_{1}$ & - & yes & - & 40 & 21 & 6.0 & - & - & - & - & 14 & 263 & 266 & 0.97 \\
\hline & & - & $\mathrm{hWG}_{2}$ & - & yes & - & 40 & 21 & 6.0 & - & - & - & - & 14 & 248 & 254 & 0.97 \\
\hline & SDAP & 2.5 & $\begin{array}{c}\text { SBM46 \& } \\
\text { hWG }_{1}\end{array}$ & - & yes & - & 40 & 21 & 6.0 & - & - & - & - & 14 & 288 & 290 & 0.94 \\
\hline & SDAP & 5 & $\begin{array}{c}\mathrm{SBM} 46 \text { \& } \\
\mathrm{hWG}_{2}\end{array}$ & - & yes & - & 40 & 21 & 6.0 & - & - & - & - & 14 & 298 & 309 & 0.97 \\
\hline Lawrence et al. (2004) & SDAP & 6 & $\mathrm{WG}_{1} \& \mathrm{WG}_{2}$ & - & yes & - & 44 & 21 & 6.1 & - & - & - & - & 21 & 413 & 468 & 1.08 \\
\hline & SDAP & 3 & $\mathrm{WG}_{1}$ & - & yes & - & 44 & 21 & 6.1 & - & - & - & - & 21 & 377 & 419 & 1.06 \\
\hline & & - & $\mathrm{WG}_{1}$ & - & yes & - & 44 & 21 & 6.1 & - & - & - & - & 21 & 353 & 383 & 1.04 \\
\hline & SDAP & 3 & $\mathrm{WG}_{2}$ & - & yes & - & 44 & 21 & 6.1 & - & - & - & - & 21 & 394 & 439 & 1.06 \\
\hline & & - & $\mathrm{WG}_{2}$ & - & yes & - & 44 & 21 & 6.1 & - & - & - & - & 21 & 363 & 392 & 1.03 \\
\hline Owusu-Asiedu et al. (2003a) & & - & PPI & - & - & yes & 18 & 10 & 3.5 & 7 & 46 & 64 & 1.39 & 14 & 85 & 114 & 1.35 \\
\hline & & - & PPI & Egg yolk AB & - & yes & 18 & 10 & 3.5 & 7 & 72 & 95 & 1.31 & 14 & 123 & 157 & 1.27 \\
\hline & SDPP & 10 & PPI & - & - & yes & 18 & 10 & 3.5 & 7 & 78 & 102 & 1.31 & 14 & 127 & 167 & 1.31 \\
\hline & SDPP & 10 & PPI & Egg yolk AB & - & yes & 18 & 10 & 3.5 & 7 & 78 & 103 & 1.32 & 14 & 130 & 174 & 1.34 \\
\hline & SDPP & 5 & PPI & - & - & yes & 24 & 10 & 3.5 & 7 & 83 & 109 & 1.31 & 14 & 132 & 173 & 1.31 \\
\hline Owusu-Asiedu et al. (2003b) & SDPP & 10 & PPI & - & - & yes & 15 & 10 & 3.8 & 7 & 138 & 187 & 1.36 & 14 & 157 & 213 & 1.36 \\
\hline & & - & PPI & - & - & yes & 15 & 10 & 3.8 & 7 & 100 & 147 & 1.47 & 14 & 101 & 141 & 1.40 \\
\hline & & - & PPI & Egg yolk AB & - & yes & 15 & 10 & 3.8 & 7 & 134 & 186 & 1.39 & 14 & 151 & 208 & 1.38 \\
\hline & & - & PPI & $\mathrm{ZnO}$ & - & yes & 15 & 10 & 3.8 & 7 & 129 & 173 & 1.34 & 14 & 159 & 215 & 1.35 \\
\hline & & - & PPI & Fumaric acid & - & yes & 15 & 10 & 3.8 & 7 & 126 & 177 & 1.40 & 14 & 155 & 212 & 1.36 \\
\hline & & - & PPI & Carbadox & - & yes & 15 & 10 & 3.8 & 7 & 128 & 184 & 1.44 & 14 & 153 & 222 & 1.46 \\
\hline Torrallardona et al. (2003) & & - & FM & - & no & yes & 12 & 24 & 7.1 & 7 & 24 & 164 & 7.69 & 14 & 101 & 227 & 2.33 \\
\hline & & - & FM & Colistin & yes & yes & 12 & 24 & 7.1 & 7 & 122 & 198 & 1.67 & 14 & 214 & 298 & 1.41 \\
\hline & SDAP & 7 & FM & - & no & yes & 12 & 24 & 7.1 & 7 & 142 & 212 & 1.47 & 14 & 193 & 268 & 1.39 \\
\hline & SDAP & 7 & FM & Colistin & yes & yes & 12 & 24 & 7.1 & 7 & 140 & 232 & 1.64 & 14 & 230 & 286 & 1.23 \\
\hline Owusu-Asiedu et al. (2002) & SDPP & 10 & SDPPa & - & no & - & 24 & 10 & 3.2 & 7 & 66 & 100 & 1.51 & 14 & 127 & 185 & 1.46 \\
\hline & SDPP & 10 & SDPPa & Egg yolk AB & no & - & 24 & 10 & 3.2 & 7 & 81 & 119 & 1.48 & 14 & 140 & 198 & 1.41 \\
\hline & & - & SDPPa & - & no & - & 24 & 10 & 3.2 & 7 & 61 & 90 & 1.48 & 14 & 102 & 152 & 1.48 \\
\hline & & - & SDPPa & Egg yolk AB & no & - & 24 & 10 & 3.2 & 7 & 72 & 104 & 1.44 & 14 & 121 & 178 & 1.46 \\
\hline Owusu-Asiedu et al. (2002) & & - & SDAP & - & no & yes & 18 & 10 & 3.5 & 7 & 74 & 107 & 1.45 & 14 & 146 & 192 & 1.31 \\
\hline & & - & SDAP & Egg yolk AB & no & yes & 18 & 10 & 3.5 & 7 & 84 & 122 & 1.45 & 14 & 175 & 230 & 1.31 \\
\hline & SDPP & 10 & SDAP & - & no & yes & 18 & 10 & 3.5 & 7 & 91 & 136 & 1.48 & 14 & 176 & 235 & 1.33 \\
\hline & SDPP & 10 & SDAP & Egg yolk AB & no & yes & 18 & 10 & 3.5 & 7 & 97 & 143 & 1.47 & 14 & 180 & 237 & 1.32 \\
\hline Torrallardona et al. (2002) & & - & FM & - & no & - & 16 & 22 & 4.8 & - & - & - & - & 14 & 206 & 273 & 1.33 \\
\hline & SDAP & 5 & FM & - & no & - & 16 & 22 & 4.8 & - & - & - & - & 14 & 227 & 272 & 1.20 \\
\hline & & - & FM & Colistin & yes & - & 16 & 22 & 4.8 & - & - & - & - & 14 & 216 & 271 & 1.27 \\
\hline & SDAP & 5 & FM & Colistin & yes & - & 16 & 22 & 4.8 & - & - & - & - & 14 & 212 & 260 & 1.23 \\
\hline & & - & FM & - & no & - & 16 & 32 & 6.4 & - & - & - & - & 14 & 334 & 423 & 1.26 \\
\hline & SDAP & 5 & FM & - & no & - & 16 & 32 & 6.4 & - & - & - & - & 14 & 336 & 420 & 1.26 \\
\hline & & - & FM & Colistin & yes & - & 16 & 32 & 6.4 & - & - & - & - & 14 & 360 & 442 & 1.23 \\
\hline & SDAP & 5 & FM & Colistin & yes & - & 16 & 32 & 6.4 & - & - & - & - & 14 & 368 & 446 & 1.21 \\
\hline Torrallardona et al. (2002) & & - & FM & - & no & - & 24 & 21 & 5.7 & - & - & - & - & 14 & 151 & 226 & 1.44 \\
\hline & SDAP & 5 & FM & - & no & - & 24 & 21 & 5.7 & - & - & - & - & 14 & 202 & 254 & 1.31 \\
\hline & & - & FM & Colistin & yes & - & 24 & 21 & 5.7 & - & - & - & - & 14 & 178 & 234 & 1.31 \\
\hline & SDAP & 5 & FM & Colistin & yes & - & 24 & 21 & 5.7 & - & - & - & - & 14 & 210 & 243 & 1.19 \\
\hline Van Dijk et al. (2002a) & & - & SBM+WHY & - & no & yes & 10 & 19 & 6.6 & 6 & -46 & - & - & 14 & -47 & - & - \\
\hline & SDPP & 8 & SBM+WHY & - & no & yes & 10 & 19 & 7.2 & 6 & -1 & - & - & 14 & 42 & - & - \\
\hline Van Dijk et al. (2002b) & & - & $\mathrm{FM}+\mathrm{DSM}$ & - & no & - & 80 & 26 & 8.0 & 7 & 176 & 185 & 1.06 & 21 & 339 & 421 & 1.24 \\
\hline & SDPP & 3 & $\mathrm{FM}+\mathrm{DSM}$ & - & no & - & 80 & 26 & 8.0 & 7 & 196 & 194 & 1.02 & 21 & 361 & 434 & 1.20 \\
\hline Van Dijk et al. (2002b) & & - & $\mathrm{FM}+\mathrm{WHY}$ & - & no & - & 88 & 26 & 8.0 & - & - & - & - & 21 & 316 & 409 & 1.30 \\
\hline & SDPP & 3 & WHY & - & no & - & 88 & 26 & 8.0 & - & - & - & - & 21 & 314 & 416 & 1.32 \\
\hline & SDPP & 3 & FM & - & no & - & 88 & 26 & 8.0 & - & - & - & - & 21 & 331 & 421 & 1.27 \\
\hline
\end{tabular}


Table 2. Summary of trials testing spray dried plasma as a protein source for piglets at weaning (Continued)

\begin{tabular}{|c|c|c|c|c|c|c|c|c|c|c|c|c|c|c|c|c|c|}
\hline \multirow{2}{*}{ Ref. } & \multirow{2}{*}{ Plasma } & \multirow{2}{*}{ Dose } & \multirow{2}{*}{ Ref. protein } & \multirow{2}{*}{$\begin{array}{l}\text { Alternative } \\
\text { product }\end{array}$} & Medication & Challenøe & Pios/tr & Age & Weight & & First & t week & & & Pre-sta & rter pha & \\
\hline & & & & & VIe & Cindnenge & Figs/u & Age & vieignt & $\mathrm{d}$ & ADG & ADFI & FGR & $\mathrm{d}$ & ADG & ADFI & FGR \\
\hline Bosi et al. (2001) & & - & CAS & - & no & yes & 12 & 19 & 4.9 & - & - & - & - & 15 & 101 & 226 & 2.25 \\
\hline & SDPP & 25 & CAS & - & no & yes & 12 & 19 & 4.9 & - & - & - & - & 15 & 100 & 251 & 2.52 \\
\hline & SDAP & 25 & CAS & - & no & yes & 12 & 19 & 4.9 & - & - & - & - & 15 & 139 & 251 & 1.80 \\
\hline & SDAP & 25 & CAS & - & no & yes & 12 & 19 & 4.9 & - & - & - & - & 15 & 169 & 252 & 1.49 \\
\hline Bosi et al. (2001) & & - & CAS & - & no & yes & 12 & 13 & 4.2 & - & - & - & - & 14 & 100 & 197 & 1.96 \\
\hline & SDPP & 25 & CAS & - & no & yes & 12 & 13 & 4.2 & - & - & - & - & 14 & 118 & 208 & 1.78 \\
\hline & SDAP & 25 & CAS & - & no & yes & 12 & 13 & 4.2 & - & - & - & - & 14 & 107 & 208 & 1.96 \\
\hline & SDAP & 25 & CAS & - & no & yes & 12 & 13 & 4.2 & - & - & - & - & 14 & 140 & 215 & 1.53 \\
\hline Grinstead et al. (2000) & & - & SBM48 & - & yes & - & 36 & 17 & 5.1 & 7 & 173 & 223 & 1.30 & 14 & 228 & 301 & 1.32 \\
\hline & SDAP & 2.5 & $\begin{array}{l}\text { SBM48 \& } \\
\text { WHY }_{1}\end{array}$ & - & yes & - & 36 & 17 & 5.1 & 7 & 214 & 238 & 1.11 & 14 & 262 & 316 & 1.20 \\
\hline & SDAP & 5 & $\begin{array}{c}\text { SBM48 \& } \\
\text { WHY }_{2}\end{array}$ & - & yes & - & 36 & 17 & 5.1 & 7 & 243 & 267 & 1.10 & 14 & 290 & 341 & 1.18 \\
\hline & & - & $\mathrm{WHY}_{1}$ & - & yes & - & 36 & 17 & 5.1 & 7 & 223 & 235 & 1.05 & 14 & 267 & 316 & 1.18 \\
\hline & & - & $\mathrm{WHY}_{2}$ & - & yes & - & 36 & 17 & 5.1 & 7 & 225 & 237 & 1.04 & 14 & 277 & 305 & 1.11 \\
\hline Grinstead et al. (2000) & SDAP & 6.7 & WHY & - & yes & - & 62 & 12 & 4.2 & 7 & 116 & 131 & 1.12 & 14 & 163 & 193 & 1.18 \\
\hline & SDAP & 5 & WHY & - & yes & - & 62 & 12 & 4.2 & 7 & 127 & 144 & 1.14 & 14 & 178 & 210 & 1.19 \\
\hline & SDAP & 3.35 & WHY & - & yes & - & 62 & 12 & 4.2 & 7 & 130 & 137 & 1.05 & 14 & 182 & 195 & 1.08 \\
\hline & SDAP & 1.7 & WHY & - & yes & - & 62 & 12 & 4.2 & 7 & 122 & 137 & 1.06 & 14 & 183 & 215 & 1.18 \\
\hline & & - & WHY & - & yes & - & 62 & 12 & 4.2 & 7 & 112 & 123 & 1.16 & 14 & 169 & 197 & 1.16 \\
\hline Grinstead et al. (2000) & & - & DSM & - & yes & - & 36 & 19 & 5.8 & 7 & 190 & 173 & 0.91 & 14 & 277 & 278 & 1.01 \\
\hline & SDAP & 2.5 & $\mathrm{DSM}_{2} \mathrm{WHY}_{1}$ & - & yes & - & 36 & 19 & 5.8 & 7 & 219 & 203 & 0.93 & 14 & 289 & 280 & 0.96 \\
\hline & SDAP & 5 & DSM \& WHY 2 & - & yes & - & 36 & 19 & 5.8 & 7 & 239 & 222 & 0.93 & 14 & 278 & 290 & 1.04 \\
\hline & & - & $\mathrm{WHY}_{1}$ & - & yes & - & 36 & 19 & 5.8 & 7 & 196 & 190 & 0.96 & 14 & 281 & 279 & 0.99 \\
\hline & & - & $\mathrm{WHY}_{2}$ & - & yes & - & 36 & 19 & 5.8 & 7 & 195 & 185 & 0.95 & 14 & 289 & 281 & 0.97 \\
\hline Chae et al. (1999) & & - & SBM48 & - & yes & - & 30 & 22 & 6.7 & 7 & 103 & 176 & 1.76 & 7 & 103 & 176 & 1.76 \\
\hline & & - & DSM & - & yes & - & 30 & 22 & 6.7 & 7 & 160 & 217 & 1.30 & 7 & 160 & 217 & 1.30 \\
\hline & & - & iSP & - & yes & - & 30 & 22 & 6.7 & 7 & 113 & 208 & 1.82 & 7 & 113 & 208 & 1.82 \\
\hline & SDAP & 18.86 & All others & - & yes & - & 30 & 22 & 6.7 & 7 & 255 & 313 & 1.23 & 7 & 255 & 313 & 1.23 \\
\hline & & - & WG & - & yes & - & 30 & 22 & 6.7 & 7 & 180 & 248 & 1.39 & 7 & 180 & 248 & 1.39 \\
\hline Angulo and Cubiló (1998) & SDPP & 6 & SBM55 & - & yes & - & 60 & 22 & 6.2 & 7 & - & 300 & - & 14 & 263 & 338 & 1.29 \\
\hline & SDPP & 3 & SBM55 & - & yes & - & 60 & 22 & 6.2 & 7 & - & 266 & - & 14 & 220 & 293 & 1.32 \\
\hline & & - & SBM55 & - & yes & - & 60 & 22 & 6.2 & 7 & - & 211 & - & 14 & 190 & 253 & 1.34 \\
\hline Kerr et al. (1998) & SDAP & 7 & PP & - & yes & - & 36 & 20 & 5.9 & 7 & 245 & 263 & 1.10 & 14 & 290 & 349 & 1.20 \\
\hline & SDAP & 5.25 & PP & - & yes & - & 36 & 20 & 5.9 & 7 & 308 & 308 & 1.00 & 14 & 349 & 386 & 1.12 \\
\hline & SDAP & 3.5 & PP & - & yes & - & 36 & 20 & 5.9 & 7 & 295 & 277 & 0.94 & 14 & 322 & 349 & 1.09 \\
\hline & SDAP & 1.75 & PP & - & yes & - & 36 & 20 & 5.9 & 7 & 308 & 299 & 0.98 & 14 & 336 & 376 & 1.12 \\
\hline & & - & PP & - & yes & - & 36 & 20 & 5.9 & 7 & 263 & 254 & 0.99 & 14 & 331 & 340 & 1.03 \\
\hline Kerr et al. (1998) & & - & DSM & - & yes & - & 35 & 20 & 5.5 & 7 & 286 & 240 & 1.18 & 14 & 376 & 386 & 1.02 \\
\hline & SDAP & 3.5 & $\mathrm{DSM} \& \mathrm{PP}_{1}$ & - & yes & - & 35 & 20 & 5.5 & 7 & 304 & 277 & 1.09 & 14 & 367 & 376 & 1.03 \\
\hline & SDAP & 7 & $\mathrm{DSM} \& \mathrm{PP}_{2}$ & - & yes & - & 35 & 20 & 5.5 & 7 & 331 & 304 & 1.10 & 14 & 376 & 395 & 1.05 \\
\hline & & - & $\mathrm{PP}_{1}$ & - & yes & - & 35 & 20 & 5.5 & 7 & 295 & 272 & 1.08 & 14 & 390 & 403 & 1.03 \\
\hline & & - & $\mathrm{PP}_{2}$ & - & yes & - & 35 & 20 & 5.5 & 7 & 240 & 240 & 1.00 & 14 & 349 & 372 & 1.06 \\
\hline Cain and Zimmerman (1997) & & - & CAS & - & no & - & 8 & 12 & 5.0 & - & - & - & - & 14 & 175 & 330 & 1.51 \\
\hline & SDAP & 6 & CAS & - & no & - & 8 & 12 & 5.0 & - & - & - & - & 14 & 164 & 360 & 1.62 \\
\hline Nessmith et al. (1997) & & - & $\mathrm{FM}+\mathrm{CAS}$ & - & - & - & 180 & 19 & 5.3 & 7 & 225 & 190 & 0.84 & 14 & 316 & 290 & 0.92 \\
\hline & SDAP & 7.5 & $\mathrm{FM}+\mathrm{CAS}$ & - & - & - & 180 & 19 & 5.3 & 7 & 262 & 227 & 0.87 & 14 & 332 & 317 & 0.95 \\
\hline Nessmith et al. (1997) & & - & SPC & - & - & - & 162 & 10 & 3.7 & 5 & 106 & 95 & 0.88 & 10 & 165 & 160 & 0.98 \\
\hline & SDAP & 7 & SPC & - & - & - & 162 & 10 & 3.7 & 5 & 129 & 105 & 0.83 & 10 & 182 & 182 & 1.00 \\
\hline Peet Schwering et al. (1997) & & - & FM & - & no & - & 360 & 28 & 7.6 & 8 & 195 & 230 & 1.23 & 8 & 195 & 230 & 1.23 \\
\hline & SDAP & 5 & FM & - & no & - & 360 & 28 & 7.6 & 8 & 232 & 250 & 1.11 & 8 & 232 & 250 & 1.11 \\
\hline Woodgate et al. (1997) & SDAP & 6 & UP1673 & - & - & - & 36 & 19 & 5.5 & - & - & - & - & 21 & 276 & 359 & 1.30 \\
\hline & & - & UP1673 & - & - & - & 36 & 19 & 5.5 & - & - & - & - & 21 & 276 & 369 & 1.34 \\
\hline Burnham et al. (1995) & SDPP & 8 & WG & - & - & - & 30 & - & 5.6 & - & - & - & - & 14 & 412 & 433 & 1.05 \\
\hline & SDPP & 6 & WG & - & - & - & 30 & - & 5.6 & - & - & - & - & 14 & 427 & 457 & 1.07 \\
\hline & SDPP & 4 & WG & - & - & - & 30 & - & 5.6 & - & - & - & - & 14 & 426 & 458 & 1.07 \\
\hline & SDPP & 2 & WG & - & - & - & 30 & - & 5.6 & - & - & - & - & 14 & 393 & 435 & 1.11 \\
\hline & & - & WG & - & - & - & 30 & - & 5.6 & - & - & - & - & 14 & 357 & 393 & 1.09 \\
\hline Coffey and Cromwell (1995) & & - & DSM & - & yes & - & 16 & 17 & 4.9 & - & - & - & - & 14 & 310 & 497 & 1.60 \\
\hline & SDPP & 3 & DSM & - & yes & - & 16 & 17 & 4.9 & - & - & - & - & 14 & 313 & 488 & 1.56 \\
\hline & SDPP & 6 & DSM & - & yes & - & 16 & 17 & 4.9 & - & - & - & - & 14 & 287 & 512 & 1.79 \\
\hline & SDPP & 9 & DSM & - & yes & - & 16 & 17 & 4.9 & - & - & - & - & 14 & 289 & 532 & 1.85 \\
\hline & SDPP & 12 & DSM & - & yes & - & 16 & 17 & 4.9 & - & - & - & - & 14 & 268 & 500 & 1.87 \\
\hline Coffey and Cromwell (1995) & & - & DSM & - & yes & - & 20 & 18 & 5.4 & - & - & - & - & 14 & 263 & 402 & 1.53 \\
\hline & SDPP & 8.3 & DSM & - & yes & - & 20 & 18 & 5.4 & - & - & - & - & 14 & 277 & 470 & 1.70 \\
\hline & & - & DSM & - & yes & yes & 20 & 18 & 5.4 & - & - & - & - & 14 & 214 & 251 & 1.18 \\
\hline & SDPP & 8.3 & DSM & - & yes & yes & 20 & 18 & 5.4 & - & - & - & - & 14 & 300 & 399 & 1.34 \\
\hline Coffey and Cromwell (1995) & & - & DSM & - & yes & - & 20 & 18 & 5.4 & - & - & - & - & 14 & 304 & 419 & 1.39 \\
\hline & SDPP & 8.3 & DSM & - & yes & - & 20 & 18 & 5.4 & - & - & - & - & 14 & 323 & 496 & 1.54 \\
\hline & & - & DSM & - & yes & yes & 20 & 18 & 5.4 & - & - & - & - & 14 & 192 & 269 & 1.43 \\
\hline & SDPP & 8.3 & DSM & - & yes & yes & 20 & 18 & 5.4 & - & - & - & - & 14 & 238 & 352 & 1.48 \\
\hline
\end{tabular}


Table 2. Summary of trials testing spray dried plasma as a protein source for piglets at weaning (Continued)

\begin{tabular}{|c|c|c|c|c|c|c|c|c|c|c|c|c|c|c|c|c|c|}
\hline \multirow{2}{*}{ Ref. } & \multirow{2}{*}{ Plasma } & \multirow{2}{*}{ Dose } & \multirow{2}{*}{ Ref. protein } & \multirow{2}{*}{$\begin{array}{c}\text { Alternative } \\
\text { product }\end{array}$} & Modication & Challonge & Piøc/tr & A & Whight & & First & t week & & & Pre-sta & irter pha & \\
\hline & & & & & Mrencatuon & Crlanenge & $\mathrm{PIgS/LI}$ & Age & vveignt & $\mathrm{d}$ & ADG & ADFI & $\overline{F G R}$ & $\mathrm{~d}$ & ADG & ADFI & FGR \\
\hline Coffey and Cromwell (1995) & & - & SBM48 & $\mathrm{Cu}+$ Antibiotics & yes & - & 24 & 30 & 7.3 & - & - & - & - & 14 & 203 & 317 & 1.57 \\
\hline & & - & DSM & - & no & - & 24 & 30 & 7.3 & - & - & - & - & 14 & 155 & 249 & 1.61 \\
\hline & & - & DSM & $\mathrm{Cu}+$ Antibiotics & yes & - & 24 & 30 & 7.3 & - & - & - & - & 14 & 244 & 363 & 1.49 \\
\hline & SDPP & 5 & DSM & - & no & - & 24 & 30 & 7.3 & - & - & - & - & 14 & 237 & 391 & 1.65 \\
\hline & SDPP & 5 & SBM48 \& DSM & $\mathrm{Cu}+$ Antibiotics & yes & - & 24 & 30 & 7.3 & - & - & - & - & 14 & 343 & 488 & 1.42 \\
\hline de Rodas et al. (1995) & & - & DSM & - & - & - & 48 & 24 & 7.2 & 7 & 210 & 210 & 1.03 & 14 & 280 & 330 & 1.12 \\
\hline & SDPP & 4 & SDBM \& DSM & - & - & - & 48 & 24 & 7.2 & 7 & 280 & 270 & 0.98 & 14 & 360 & 410 & 1.11 \\
\hline & & - & SDBM & - & - & - & 48 & 24 & 7.2 & 7 & 250 & 240 & 0.97 & 14 & 320 & 370 & 1.10 \\
\hline de Rodas et al. (1995) & & - & SBM44 & - & - & - & 9 & 20 & 6.1 & 7 & 160 & 310 & 2.04 & 14 & 280 & 420 & 1.59 \\
\hline & SDPP & 14 & SBM44 & - & - & - & 9 & 20 & 6.1 & 7 & 250 & 390 & 1.56 & 14 & 360 & 510 & 1.45 \\
\hline Gatnau et al. (1995) & & - & CAS & - & no & yes & 27 & 19 & 6.0 & - & - & - & - & 15 & 19 & 181 & 7.75 \\
\hline & SDPP & 8 & CAS & - & no & yes & 27 & 19 & 6.0 & - & - & - & - & 15 & 134 & 262 & 3.11 \\
\hline & & - & CAS & Albumin & no & yes & 27 & 19 & 6.0 & - & - & - & - & 15 & 78 & 244 & 4.61 \\
\hline & & - & CAS & IgG & no & yes & 27 & 19 & 6.0 & - & - & - & - & 15 & 158 & 273 & 2.93 \\
\hline & & - & CAS & LMW & no & yes & 27 & 19 & 6.0 & - & - & - & - & 15 & 50 & 191 & 4.69 \\
\hline Nessmith Jr et al. (1995) & SDAP & 7 & SDEP & - & - & - & 54 & 14 & 4.3 & - & - & - & - & 14 & 209 & - & 1.19 \\
\hline & SDAP & 5.25 & SDEP & - & - & - & 54 & 14 & 4.3 & - & - & - & - & 14 & 203 & - & 1.19 \\
\hline & SDAP & 3.5 & SDEP & - & - & - & 54 & 14 & 4.3 & - & - & - & - & 14 & 209 & - & 1.23 \\
\hline & SDAP & 1.75 & SDEP & - & - & - & 54 & 14 & 4.3 & - & - & - & - & 14 & 187 & - & 1.30 \\
\hline & & - & SDEP & - & - & - & 54 & 14 & 4.3 & - & - & - & - & 14 & 192 & - & 1.30 \\
\hline Owen et al. (1995) & & - & DSM & - & - & - & 36 & 21 & 4.5 & - & - & - & - & 14 & 237 & - & 1.03 \\
\hline & SDPP & 7.5 & DSM & - & - & - & 36 & 21 & 4.5 & - & - & - & - & 14 & 278 & - & 1.15 \\
\hline & & - & DSM & LMW & - & - & 36 & 21 & 4.5 & - & - & - & - & 14 & 251 & - & 1.01 \\
\hline & & - & DSM & IgG & - & - & 36 & 21 & 4.5 & - & - & - & - & 14 & 299 & - & 1.05 \\
\hline & & - & DSM & Albumin & - & - & 36 & 21 & 4.5 & - & - & - & - & 14 & 261 & - & 1.09 \\
\hline Owen et al. (1995) & & - & DSM & - & - & - & 42 & 10 & 3.2 & - & - & - & - & 21 & 204 & - & 1.23 \\
\hline & SDPP & 10 & DSM & - & - & - & 42 & 10 & 3.2 & - & - & - & - & 21 & 227 & - & 1.19 \\
\hline & & - & DSM & IgG & - & - & 42 & 10 & 3.2 & - & - & - & - & 21 & 254 & - & 1.14 \\
\hline & & - & DSM & Albumin & - & - & 42 & 10 & 3.2 & - & - & - & - & 21 & 231 & - & 1.15 \\
\hline Smith II et al. (1995) & & - & DSM & - & - & - & 104 & 15 & 4.3 & - & - & - & - & 14 & 191 & 248 & 1.30 \\
\hline & SDBP & 5 & DSM & - & - & - & 104 & 15 & 4.3 & - & - & - & - & 14 & 209 & 265 & 1.27 \\
\hline & SDPP & 5 & DSM & - & - & - & 104 & 15 & 4.3 & - & - & - & - & 14 & 232 & 267 & 1.15 \\
\hline Stahly et al. (1995) & SDAP & 6 & SBM & - & - & yes & 20 & 19 & 5.9 & - & - & - & - & 20 & 536 & 327 & 1.66 \\
\hline & & - & SBM & - & - & yes & 20 & 19 & 5.9 & - & - & - & - & 20 & 450 & 245 & 1.88 \\
\hline & SDAP & 6 & SBM & - & - & - & 20 & 19 & 5.9 & - & - & - & - & 20 & 582 & 409 & 1.42 \\
\hline & & - & SBM & - & - & - & 20 & 19 & 5.9 & - & - & - & - & 20 & 573 & 400 & 1.44 \\
\hline Stahly et al. (1995) & SDIPP & 4 & CAS & - & - & - & 10 & 20 & 6.0 & - & - & - & - & 15 & 227 & 311 & 1.36 \\
\hline & SDPP & 4 & CAS & - & - & - & 10 & 20 & 6.0 & - & - & - & - & 15 & 241 & 321 & 1.33 \\
\hline & & - & CAS & - & - & - & 10 & 20 & 6.0 & - & - & - & - & 20 & 178 & 304 & 1.69 \\
\hline Weaver et al. (1995) & & - & CAS & - & - & - & 20 & - & 6.7 & 7 & 51 & 118 & 2.27 & 14 & 198 & 247 & 1.22 \\
\hline & SDAP & 8 & CAS & - & - & - & 20 & - & 6.7 & 7 & 115 & 184 & 1.61 & 14 & 223 & 300 & 1.37 \\
\hline & & - & CAS & LMW & - & - & 20 & - & 6.7 & 7 & 49 & 119 & 2.44 & 14 & 117 & 185 & 1.61 \\
\hline & & - & CAS & Albumin & - & - & 20 & - & 6.7 & 7 & 103 & 170 & 1.67 & 14 & 212 & 294 & 1.39 \\
\hline & & - & CAS & IgG & - & - & 20 & - & 6.7 & 7 & 111 & 175 & 1.59 & 14 & 218 & 284 & 1.32 \\
\hline Kats et al. (1994) & & - & DSM & - & yes & - & 89 & 21 & 6.4 & - & - & - & - & 14 & 165 & 206 & 1.27 \\
\hline & SDPP & 2 & DSM & - & yes & - & 89 & 21 & 6.4 & - & - & - & - & 14 & 206 & 244 & 1.19 \\
\hline & SDPP & 4 & DSM & - & yes & - & 89 & 21 & 6.4 & - & - & - & - & 14 & 217 & 256 & 1.18 \\
\hline & SDPP & 6 & DSM & - & yes & - & 89 & 21 & 6.4 & - & - & - & - & 14 & 240 & 290 & 1.22 \\
\hline & SDPP & 8 & DSM & - & yes & - & 89 & 21 & 6.4 & - & - & - & - & 14 & 247 & 302 & 1.23 \\
\hline & SDPP & 10 & DSM & - & yes & - & 89 & 21 & 6.4 & - & - & - & - & 14 & 255 & 300 & 1.19 \\
\hline Kats et al. (1994) & SDPP & 10 & SDBM & - & yes & - & 60 & 19 & 5.5 & - & - & - & - & 14 & 231 & 286 & 1.23 \\
\hline & SDPP & 7.5 & SDBM & - & yes & - & 60 & 19 & 5.5 & - & - & - & - & 14 & 258 & 304 & 1.16 \\
\hline & SDPP & 5 & SDBM & - & yes & - & 60 & 19 & 5.5 & - & - & - & - & 14 & 240 & 290 & 1.20 \\
\hline & SDPP & 2.5 & SDBM & - & yes & - & 60 & 19 & 5.5 & - & - & - & - & 14 & 245 & 281 & 1.15 \\
\hline & & - & SDBM & - & yes & - & 60 & 19 & 5.5 & - & - & - & - & 14 & 231 & 281 & 1.22 \\
\hline Rantanen et al. (1994) & & - & DSM & - & - & - & 63 & 13 & 4.1 & 7 & 91 & 150 & 3.57 & 14 & 163 & 186 & 2.50 \\
\hline & $\mathrm{SDPP}_{1}$ & 2 & DSM & - & - & - & 63 & 13 & 4.1 & 7 & 124 & 175 & 2.94 & 14 & 195 & 217 & 2.44 \\
\hline & $\mathrm{SDPP}_{1}$ & 4 & DSM & - & - & - & 63 & 13 & 4.1 & 7 & 123 & 176 & 3.13 & 14 & 204 & 234 & 2.56 \\
\hline & $\mathrm{SDPP}_{1}$ & 6 & DSM & - & - & - & 63 & 13 & 4.1 & 7 & 145 & 200 & 2.94 & 14 & 212 & 236 & 2.44 \\
\hline & $\mathrm{SDPP}_{2}$ & 2 & DSM & - & - & - & 63 & 13 & 4.1 & 7 & 124 & 177 & 3.03 & 14 & 195 & 222 & 2.50 \\
\hline & $\mathrm{SDPP}_{2}$ & 4 & DSM & - & - & - & 63 & 13 & 4.1 & 7 & 136 & 186 & 2.86 & 14 & 215 & 237 & 2.44 \\
\hline & $\mathrm{SDPP}_{2}$ & 6 & DSM & - & - & - & 63 & 13 & 4.1 & 7 & 150 & 195 & 2.86 & 14 & 209 & 241 & 2.56 \\
\hline & SDBP & 2 & DSM & - & - & - & 63 & 13 & 4.1 & 7 & 109 & 173 & 3.23 & 14 & 182 & 204 & 2.50 \\
\hline & SDBP & 4 & DSM & - & - & - & 63 & 13 & 4.1 & 7 & 141 & 186 & 2.86 & 14 & 204 & 219 & 2.38 \\
\hline & SDBP & 6 & DSM & - & - & - & 63 & 13 & 4.1 & 7 & 145 & 182 & 2.5 & 14 & 200 & 227 & 2.50 \\
\hline Rojas et al. (1994) & & - & SBM48 & - & no & - & 20 & 21 & 5.9 & - & - & - & - & 14 & 64 & 218 & 3.45 \\
\hline & SDPP & 10 & SBM48 & - & no & - & 20 & 21 & 5.9 & - & - & - & - & 14 & 218 & 358 & 1.64 \\
\hline & & - & SBM48 & $\mathrm{Cu}+$ Antibiotics & yes & - & 20 & 21 & 5.9 & - & - & - & - & 14 & 145 & 277 & 1.92 \\
\hline & SDPP & 10 & SBM48 & $\mathrm{Cu}+$ Antibiotics & yes & - & 20 & 21 & 5.9 & - & - & - & - & 14 & 304 & 449 & 1.47 \\
\hline
\end{tabular}


Table 2. Summary of trials testing spray dried plasma as a protein source for piglets at weaning (Continued)

\begin{tabular}{|c|c|c|c|c|c|c|c|c|c|c|c|c|c|c|c|c|c|}
\hline \multirow{2}{*}{ Ref. } & \multirow{2}{*}{ Plasma } & \multirow{2}{*}{ Dose } & \multirow{2}{*}{ Ref. Protein } & \multirow{2}{*}{$\begin{array}{l}\text { Alternative } \\
\text { Product }\end{array}$} & \multirow{2}{*}{ Medication } & \multirow{2}{*}{ Challenge } & \multirow{2}{*}{ Pigs/tr } & \multirow{2}{*}{ Age } & \multirow{2}{*}{ Weight } & \multicolumn{4}{|c|}{ First week } & \multicolumn{4}{|c|}{ Pre-starter phase } \\
\hline & & & & & & & & & & $\mathrm{d}$ & ADG & ADFI & $\overline{F G R}$ & $\mathrm{~d}$ & ADG & ADFI & FGR \\
\hline \multirow[t]{6}{*}{ Hansen et al. (1993) } & & - & DSM & - & yes & - & 30 & 21 & 5.9 & 7 & 306 & 299 & 0.97 & 14 & 315 & 389 & 1.23 \\
\hline & SDPP & 10.35 & DSM \& SBM48 & - & yes & - & 30 & 21 & 5.9 & 7 & 402 & 398 & 0.99 & 14 & 444 & 537 & 1.20 \\
\hline & SDPP & 10.35 & DSM \& SBM48 & - & yes & - & 30 & 21 & 5.9 & 7 & 374 & 346 & 0.92 & 14 & 420 & 487 & 1.16 \\
\hline & SDPP & 13.4 & $\mathrm{DSM}+\mathrm{WHY}_{1}$ & - & yes & - & 30 & 21 & 5.9 & 7 & 371 & 362 & 0.97 & 14 & 413 & 482 & 1.16 \\
\hline & SDPP & 13.4 & $\mathrm{DSM}+\mathrm{WHY}_{2}$ & - & yes & - & 30 & 21 & 5.9 & 7 & 313 & 302 & 0.96 & 14 & 378 & 437 & 1.15 \\
\hline & & - & SBM48 & - & yes & - & 30 & 21 & 5.9 & 7 & 260 & 281 & 1.06 & 14 & 327 & 416 & 1.27 \\
\hline \multirow[t]{5}{*}{ Hansen et al. (1993) } & & - & DSM & - & yes & - & 30 & 21 & 5.3 & 7 & 321 & 309 & 0.95 & 14 & 328 & 390 & 1.18 \\
\hline & SDPP & 10.28 & All others & - & yes & - & 30 & 21 & 5.3 & 7 & 359 & 374 & 1.04 & 14 & 378 & 499 & 1.32 \\
\hline & & - & SDB & - & yes & - & 30 & 21 & 5.3 & 7 & 287 & 293 & 1.01 & 14 & 341 & 405 & 1.19 \\
\hline & & - & MEX & - & yes & - & 30 & 21 & 5.3 & 7 & 224 & 290 & 1.28 & 14 & 263 & 408 & 1.52 \\
\hline & SDBP & 6.96 & All others & - & yes & - & 30 & 21 & 5.3 & 7 & 311 & 325 & 1.03 & 14 & 327 & 422 & 1.28 \\
\hline \multirow[t]{2}{*}{ Hansen et al. (1993) } & & - & DSM+WHY & - & yes & - & 118 & 24 & 7.4 & 7 & 181 & 175 & 0.96 & 14 & 262 & 305 & 1.16 \\
\hline & SDPP & 10 & DSM+WHY & - & yes & - & 118 & 24 & 7.4 & 7 & 221 & 217 & 0.98 & 14 & 266 & 302 & 1.12 \\
\hline \multirow{6}{*}{$\begin{array}{l}\text { Gatnau and Zimmerman } \\
\text { (1992) }\end{array}$} & & - & SBM & - & - & - & 16 & 25 & 6.1 & - & - & - & - & 14 & 151 & 387 & 2.66 \\
\hline & SDPP & 2 & SBM & - & - & - & 16 & 25 & 6.1 & - & - & - & - & 14 & 150 & 447 & 2.95 \\
\hline & SDPP & 4 & SBM & - & - & - & 16 & 25 & 6.1 & - & - & - & - & 14 & 236 & 526 & 2.25 \\
\hline & SDPP & 6 & SBM & - & - & - & 16 & 25 & 6.1 & - & - & - & - & 14 & 254 & 528 & 2.07 \\
\hline & SDPP & 8 & SBM & - & - & - & 16 & 25 & 6.1 & - & - & - & - & 14 & 269 & 547 & 2.04 \\
\hline & SDPP & 10 & SBM & - & - & - & 16 & 25 & 6.1 & - & - & - & - & 14 & 188 & 445 & 2.44 \\
\hline \multirow[t]{2}{*}{ Kats et al. (1992) } & & - & eSPC & - & - & - & 234 & 21 & 6.0 & 9 & 74 & 134 & 2.08 & 9 & 74 & 134 & 2.08 \\
\hline & SDPP & 10 & eSPC & - & - & - & 234 & 21 & 6.0 & 9 & 138 & 183 & 1.35 & 9 & 138 & 183 & 1.35 \\
\hline \multirow{3}{*}{$\begin{array}{l}\text { Gatnau and Zimmerman } \\
\text { (1991) }\end{array}$} & SDPP & 10 & $\mathrm{BM}$ & - & - & - & 9 & - & 7.1 & - & - & - & - & 14 & 284 & 365 & 1.29 \\
\hline & SDPP & 5 & $\mathrm{BM}$ & - & - & - & 9 & - & 7.1 & - & - & - & - & 14 & 279 & 348 & 1.24 \\
\hline & & - & $\mathrm{BM}$ & - & - & - & 9 & - & 7.1 & - & - & - & - & 14 & 244 & 321 & 1.33 \\
\hline Gatnau and Zimmerman & & - & SBM & - & - & - & 24 & - & 7.1 & - & - & - & - & 14 & 63 & 119 & 1.85 \\
\hline & SDPP & 10 & SBM \& BM & - & - & - & 24 & - & 7.1 & - & - & - & - & 14 & 127 & 209 & 1.65 \\
\hline & SDPP & 5 & $\mathrm{BM}$ & - & - & - & 24 & - & 7.1 & - & - & - & - & 14 & 97 & 165 & 1.66 \\
\hline & & - & $\mathrm{BM}$ & - & - & - & 24 & - & 7.1 & - & - & - & - & 14 & 58 & 130 & 2.37 \\
\hline Gatnau et al. (1991) & & - & SBM & - & - & - & 9 & 28 & 7.1 & - & - & - & - & 14 & 138 & 243 & 2.10 \\
\hline & SDPP & 2 & SBM & - & - & - & 9 & 28 & 7.1 & - & - & - & - & 14 & 176 & 276 & 2.34 \\
\hline & SDPP & 4 & SBM & - & - & - & 9 & 28 & 7.1 & - & - & - & - & 14 & 203 & 294 & 1.49 \\
\hline & SDPP & 6 & SBM & - & - & - & 9 & 28 & 7.1 & - & - & - & - & 14 & 251 & 326 & 1.32 \\
\hline & SDPP & 8 & SBM & - & - & - & 9 & 28 & 7.1 & - & - & - & - & 14 & 188 & 290 & 1.63 \\
\hline Sohn et al. (1991) & & - & DSM & - & - & - & 48 & 24 & - & 7 & 210 & 210 & 1.03 & 14 & 280 & 330 & 1.16 \\
\hline & SDAP & 4 & DSM \& SDB & - & - & - & 48 & 24 & - & 7 & 280 & 270 & 0.99 & 14 & 360 & 410 & 1.15 \\
\hline & & - & SDB & - & - & - & 48 & 24 & - & 7 & 250 & 240 & 0.97 & 14 & 320 & 370 & 1.15 \\
\hline Gatnau and Zimmerman & & - & CAS & - & - & - & 9 & - & 6.9 & - & - & - & - & 14 & 247 & 292 & 1.18 \\
\hline & & - & MEX & - & - & - & 9 & - & 6.9 & - & - & - & - & 14 & 139 & 213 & 1.46 \\
\hline & & - & iSP & - & - & - & 9 & - & 6.9 & - & - & - & - & 14 & 153 & 204 & 1.47 \\
\hline & SDPP & 10 & All others & - & - & - & 9 & - & 6.9 & - & - & - & - & 14 & 261 & 350 & 1.34 \\
\hline $\begin{array}{l}\text { Gatnau and Zimmerman } \\
(1990)\end{array}$ & & - & SBM & - & - & - & 12 & - & 6.9 & - & - & - & - & 14 & 191 & 267 & 1.44 \\
\hline & & - & DSM & - & - & - & 12 & - & 6.9 & - & - & - & - & 14 & 230 & 300 & 1.74 \\
\hline & SDPP & 10 & SBM \& DSM & - & - & - & 12 & - & 6.9 & - & - & - & - & 14 & 345 & 462 & 1.35 \\
\hline
\end{tabular}

Albumin = Albunim fraction of plasma; BM = Blood meal; Bov IgG = Immunoglobulins from bovine origin; CAS = Casein; DSM = Dried skimed milk; $\mathrm{eSPC}=$ Extruded soy protein concentrate; FDAP $=$ Freeze dried animal plasma; FM $=$ Fishmeal; $\mathrm{hWG}=$ Hydrolysed Wheat Gluten; IgG $=$ Immunoglobulins from plasma (\% relative to plasma source); irSDAP = Irradiated spray dried animal plasma; iSP = Isolated soy protein; LMW = Low molecular fraction of plasma; $\mathrm{MEX}=$ Meat extract; $\mathrm{PP}=$ Potato protein; $\mathrm{PPI}=$ Pea protein isolate; $\mathrm{SBM}=$ Soybean meal; SBM44 $=$ Soybean meal44\%CP; SBM46 = Soybean meal-46\%CP; SBM48 = Soybean meal-48\%CP; SBM55 = Soybean meal-55\%CP; SDAP = Spray dried animal plasma; SDB = Spray dried blood; SDBM = Spray dried blood meal; SDBP $=$ Spray dried bovine plasma; SDEP $=$ Spray dried egg protein; SDIPP = Immunised spray dried porcine plasma; SDPP = Spray dried porcine plasma; SDPPa = Autoclaved spray dried porcine plasma; SPC = Soy protrein concentrate; UP1673 = Ultimate protein 1673; WG = Wheat Gluten; WHY = Whey protein.

piglets that have been used to prepare this review. The mean differences (vs. control) in each trial for weight gain, feed intake and feed to gain due to the use of SDAP have been used to calculate the performance responses of piglets according to several factors using the GLM procedure of SAS. The mean improvements over control for each group considered were compared using Tukey's Studentized Range (Honestly Significant Difference) test and each mean value was compared to a value of zero (control) using a Student's t-test. Factors considered are: nature of protein being replaced by SDAP, source of spray dried plasma used, weaning age of the pigs, presence or absence of a pathogenic challenge and presence or absence medication in the feed, and the corresponding results are presented in Table 3 to 7 .

To study a possible relationship between the positive effects observed for the diets containing SDAP and its nutritional value, it can be of interest to compare the magnitude of the responses according to the nature of the protein being replaced. A positive response should be expected if SDAP replaced a protein sources with poorer nutritive value whereas no response or a negative effect 
Table 3. Performance improvement over the control diet of piglets fed with different sources of spray dried plasma for one or two weeks after weaning

\begin{tabular}{|c|c|c|c|c|c|c|c|c|}
\hline \multirow{2}{*}{ Source } & \multicolumn{4}{|c|}{ One week post-weaning } & \multicolumn{4}{|c|}{ Two weeks post-weaning } \\
\hline & $\mathrm{n}$ & $\Delta \mathrm{ADG}(\mathrm{g} / \mathrm{d})$ & $\triangle \mathrm{ADFI}(\mathrm{g} / \mathrm{d})$ & $\Delta$ FGR $(\mathrm{g} / \mathrm{g})$ & $\mathrm{n}$ & $\Delta \mathrm{ADG}(\mathrm{g} / \mathrm{d})$ & $\triangle \mathrm{ADFI}(\mathrm{g} / \mathrm{d})$ & $\Delta$ FGR $(\mathrm{g} / \mathrm{g})$ \\
\hline$\overline{\text { SDPP }}$ & 25 & $+62 *$ & $+60^{*}$ & -0.15 & 54 & $+55^{*}$ & $+68^{\mathrm{a}, *}$ & $-0.13^{*}$ \\
\hline SDBP & 5 & $+43^{*}$ & $+44 *$ & -0.17 & 5 & +22 & $+37^{\mathrm{ab},} *$ & +0.03 \\
\hline SDAP & 52 & $+48 *$ & $+36 *$ & $-0.21^{*}$ & 84 & $+27 *$ & $+24^{\mathrm{b}, *}$ & -0.07 \\
\hline Pooled STD & & 37.7 & 36.2 & 0.730 & & 38.5 & 38.5 & 0.449 \\
\hline
\end{tabular}

${ }^{\mathrm{a}, \mathrm{b}}$ Values in same column with different letters are significantly different as analysed by Tukey’s (HSD) test (p<0.05). n: Number of trials.

* $\mathrm{p}<0.05$. Statistical significance of improvement over controls without plasma.

should be expected if the protein source being replaced had the same or a better nutritive value, respectively. Table 4 shows the productive response to plasma according to the nature of the protein being replaced. For all the protein sources being replaced, SDAP improved weight gain and feed intake which suggests that factors other than nutritive value may also be involved in the positive effects of SDAP. It is worth noting that feed to gain ratio changed very little for most protein sources. Only the replacement by SDAP of fishmeal (during the first week post-weaning) and casein (two weeks post-weaning) resulted in significant feed to gain ratio improvements. The feed intake mediated effect supports the hypothesis of an improved palatability of the feeds containing SDAP. Ermer et al. (1994) observed that piglets had a higher preference for SDPP than for dried skim milk containing feeds. This contrasts with the observations of Torrallardona and Solà-Oriol (2009), who evaluated the pig's preference for SDPP and another fourteen additional protein sources in relation to a common reference diet. SDPP preference was intermediate among the protein sources tested and did not differ significantly from them except for potato protein that had a significantly lower preference. Numerically, fishmeal, lupine, soybean meal-44, extruded soybeans, soybean meal-48, dried porcine solubles and dried skimmed milk at $10 \%$ of inclusion had higher preference values than SDPP. The higher feed intakes observed for the diets containing plasma could also result from an improved health status and higher body weight of the piglets (Torrallardona et al., 2003). In pair fed piglets plasma has also improved performance which suggests a specific biological effect independent of feed intake (Jiang et al., 2000b).

The response of weaning piglets to spray dried plasma from porcine (SDPP), bovine (SDBP), and mixed or unknown (SDAP) origin are presented in Table 3. All three sources significantly improve the performance of the piglets. It appears that plasma from porcine origin has a higher efficacy than the other sources, although statistically significant differences are only obtained for feed intake in the $0-14 \mathrm{~d}$ period. It is possible that the specific IgG against porcine pathogens in SDPP are advantageous. This is in good agreement with studies in which the direct comparison of the two sources resulted in higher productivities for SDPP than for SDBP (Hansen et al., 1993; Gatnau and Zimmerman, 1994; Rantanen et al., 1994; Smith II et al., 1995; Pierce et al., 2005). However, higher responses for SDBP have also been reported (Russell, 1994). It has also been suggested that SDPP is better than SDAP of mixed origin (Owusu-Asiedu et al., 2002). However, in a study in which SDPP was compared with two sources of SDAP

Table 4. Performance improvement over the control diet according to the protein being replaced of piglets fed spray dried plasma for one or two weeks after weaning

\begin{tabular}{|c|c|c|c|c|c|c|c|c|}
\hline \multirow{2}{*}{ Source } & \multicolumn{4}{|c|}{ One week post-weaning } & \multicolumn{4}{|c|}{ Two weeks post-weaning } \\
\hline & $\mathrm{n}$ & $\Delta \mathrm{ADG}(\mathrm{g} / \mathrm{d})$ & $\Delta \mathrm{ADFI}(\mathrm{g} / \mathrm{d})$ & $\Delta$ FGR $(\mathrm{g} / \mathrm{g})$ & $\mathrm{n}$ & $\Delta \mathrm{ADG}(\mathrm{g} / \mathrm{d})$ & $\Delta$ ADFI (g/d) & $\Delta$ FGR $(\mathrm{g} / \mathrm{g})$ \\
\hline Meat extract & 2 & $+111^{\mathrm{a},} *$ & $+60^{*}$ & -0.25 & 2 & $+90^{\mathrm{a}, *}$ & $+53 \dagger$ & -0.22 \\
\hline Soybean meal & 25 & $+71^{\mathrm{ab}, *}$ & $+50^{*}$ & -0.14 & 32 & $+57^{\mathrm{ab}, *}$ & $+52 *$ & $-0.14 \dagger$ \\
\hline Soy protein concentrate & 9 & $+74^{\mathrm{ab},} *$ & $+92 *$ & -0.24 & 9 & $+55^{\mathrm{ab}, *}$ & $+84^{*}$ & -0.00 \\
\hline Dry skimmed milk & 11 & $+47^{\mathrm{ab},} *$ & $+52 *$ & -0.01 & 26 & $+42^{\mathrm{ab}, *}$ & $+63^{*}$ & +0.03 \\
\hline Pea protein isolate & 3 & $+25^{\mathrm{b}}$ & +29 & -0.06 & 3 & $+35^{\mathrm{ab}}$ & $+47 *$ & -0.00 \\
\hline Casein & 1 & $+37^{\mathrm{ab}}$ & +37 & +0.03 & 9 & $+32^{\mathrm{ab}, *}$ & $+28 *$ & $-0.67 *$ \\
\hline Fishmeal & 7 & $+42^{\mathrm{ab}, *}$ & +18 & $-1.32 *$ & 18 & $+34^{\mathrm{ab}, *}$ & $+17 \dagger$ & -0.14 \\
\hline Whey protein & 11 & $+22^{\mathrm{b}, *}$ & $+21 \dagger$ & -0.02 & 12 & $+24^{\mathrm{ab}, *}$ & $+20 \dagger$ & -0.00 \\
\hline Wheat gluten & 2 & $+55^{\mathrm{ab}, *}$ & +34 & -0.77 & 15 & $+22^{\mathrm{b}, *}$ & $+25^{*}$ & -0.03 \\
\hline Blood protein & 3 & $+42^{\mathrm{ab}, *}$ & $+48^{*}$ & +0.02 & 7 & $+16^{\mathrm{b}}$ & $+27 \dagger$ & +0.01 \\
\hline Potato protein & 10 & $+34^{\mathrm{ab}, *}$ & $+27 *$ & -0.03 & 10 & $+7^{\mathrm{b}}$ & +15 & +0.01 \\
\hline Pooled STD & & 33.5 & 32.7 & 0.689 & & 38.5 & 39.7 & 0.429 \\
\hline
\end{tabular}

${ }^{\mathrm{a}, \mathrm{b}}$ Values in same column with different letters are significantly different as analysed by Tukey’s (HSD) test (p<0.05). n: Number of trials.

$* \mathrm{p}<0.05 ; \dagger \mathrm{p}<0.1$. Statistical significance of improvement over controls without plasma. 

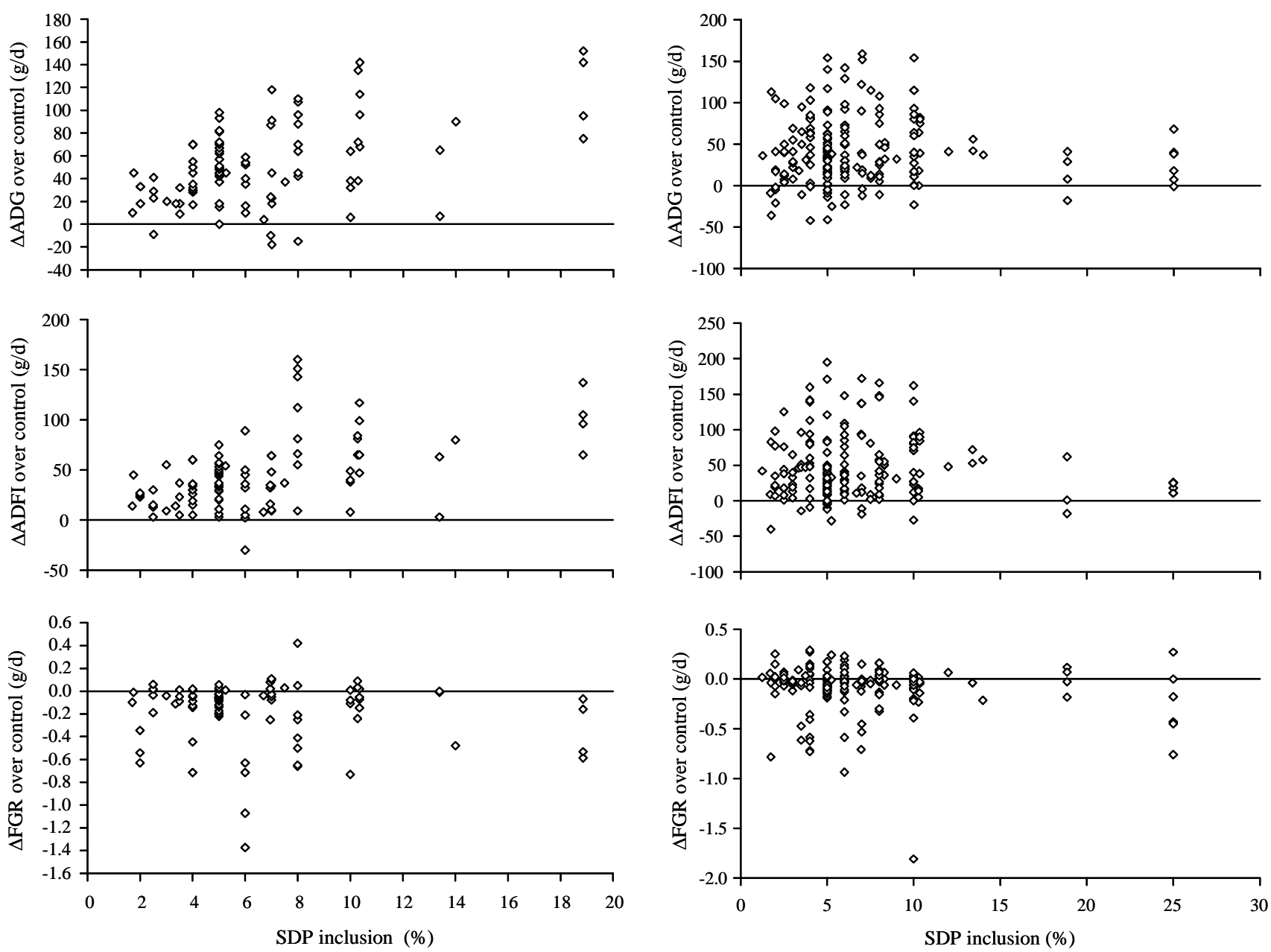

Figure 2. Effect of feeding different doses of spray dried plasma to piglets on the improvement in average daily weight gain, average daily feed intake and feed to gain ratio over the control in the first week after weaning. Number of trials (n) $=95$ for ADG, $n$ $=96$ for $\mathrm{ADFI}$ and $\mathrm{n}=92$ for FGR.

(with or without standardised IgG content), a better efficacy for the SDAP standardised in IgG content over the other sources was observed, indicating the relevance of the IgG content of the different sources of plasma (Bosi et al., 2001). The advantadge of a standardised IgG content of SDAP has also been suggested by other authors (Campbell et al., 1998; Conde, 2005). This is supported by studies in which the comparison of SDAP with its albumin, immunoglobulin and low molecular weight fractions show that the beneficial effects are associated with the immunoglobulin fraction (Gatnau et al., 1995; Owen et al., 1995; Weaver et al., 1995; Pierce et al., 2005). Autoclaving SDPP decreases efficacy and this has been explained by an inactivation of its specific antibodies (Owusu-Asiedu et al., 2002), although other bioactive components may have also been destroyed. Two SDPP sources, obtained from pigs exposed to either high or low antigenic exposure were compared, in an attempt to check if the high antigenic exposure resulted in more

Figure 3. Effect of feeding different doses of spray dried plasma to piglets on the improvement in average daily weight gain, average daily feed intake and feed to gain ratio over the control in the post-weaning phase $(0-14 \mathrm{~d})$. Number of trials $(\mathrm{n})=192$ for ADG, $n=185$ for ADFI and $n=189$ for FGR

efficient plasma, but no advantage was obtained (Stahly et al., 1995). Similarly, spray dried immune porcine plasma (SDIPP) obtained from pigs vaccinated against specific pathogens resulted in little productive advantages to piglets experimentally challenged with the same pathogen. This is probably due to an already existing "natural" antigenic load in the conventional non-vaccinated SDPP (Conde, 2005; Niewold et al., 2007)

\section{SPRAY DRIED ANIMAL PLASMA AT DIFFERENT DOSES AND AGE OF THE PIGLETS AT WEANING}

The response of piglets according to the inclusion doses of plasma in the feed is presented in Figure 2 and 3. It appears that during the first week post weaning (Figure 2) weight gain and feed intake responses to plasma increase with its level of inclusion in the feed. Over the two-week 
Table 5. Effect of weaning age on the performance improvement over the control diet of piglets fed spray dried plasma for one or two weeks after weaning

\begin{tabular}{|c|c|c|c|c|c|c|c|c|}
\hline \multirow{2}{*}{ Weaning age } & \multicolumn{4}{|c|}{ One week post-weaning } & \multicolumn{4}{|c|}{ Two weeks post-weaning } \\
\hline & $\mathrm{n}$ & $\Delta \mathrm{ADG}(\mathrm{g} / \mathrm{d})$ & $\Delta \mathrm{ADFI}(\mathrm{g} / \mathrm{d})$ & $\Delta$ FGR (g/g) & $\mathrm{n}$ & $\Delta \mathrm{ADG}(\mathrm{g} / \mathrm{d})$ & $\Delta$ ADFI (g/d) & $\Delta$ FGR (g/g) \\
\hline 10-17 days & 30 & $+43^{*}$ & $+30^{\mathrm{ab}, *}$ & -0.07 & 38 & $+25 *$ & $+27 *$ & -0.01 \\
\hline 18-24 days & 47 & $+60 *$ & $+57^{\mathrm{a}, *}$ & $-0.28 *$ & 92 & $+43^{*}$ & $+46 *$ & $-0.13^{*}$ \\
\hline 25-32 days & 6 & $+32 *$ & $+17^{\mathrm{b}}$ & -0.11 & 13 & $+38 *$ & $+42 *$ & -0.05 \\
\hline Pooled STD & & 36.9 & 34.4 & 0.722 & & 40.2 & 43.2 & 0.447 \\
\hline
\end{tabular}

pre-starter period (Figure 3), however, an optimal inclusion level of $4-8 \%$ is suggested. It is well known that during the first week post weaning piglet's feed intake is very low (Pluske et al., 1997), and therefore, the higher inclusion levels of SDAP may facilitate an adequate intake of IgG. Over the two week period, however, feed intake is normalised and the higher SDAP doses may result in nutrient imbalances (i.e. methionine, isoleucine or salt) that reduce the productive responses. Most dose-response studies have shown an optimum inclusion level for the first two weeks post-weaning, that falls within this range of 4$8 \%$. Thus, an optimum inclusion of $6 \%$ was observed compared to inclusion levels of 2 and $4 \%$ (Rantanen et al., 1994) or to inclusion levels of 2, 4 and 8\% (Gatnau et al., 1991; Burnham et al., 1995). Gatnau and Zimmerman (1992) concluded that performance was maximised at 6-8\% of inclusion after testing doses of 2, 4, 6, 8 and $10 \%$. Similarly, higher performances have been observed for 6\% over 3\% SDPP (Angulo and Cubiló, 1998; Lawrence et al., 2004), for 5\% over 2.5\% SDAP (Grinstead et al., 2000) and for $7 \%$ over $3.5 \%$ SDAP (Kerr et al., 1998). Other dose response studies, however, have shown that performance was optimised at levels of inclusion around 3\% (Coffey and Cromwell, 1995; Grinstead et al., 2000). Studies with optimised responses at higher inclusion levels have also been reported. Kats et al. (1994) demonstrated that weight gain and feed intake (but not feed efficiency) increased linearly up to inclusion levels of $10 \%$ if diets were supplemented with methionine. They concluded that supplemental methionine is required to maximise piglet performance with SDAP diets. It is likely that the maximum SDAP inclusion level will depend on the isoleucine content of the diet as this will become the most limiting amino acid after methionine supplementation (Figure 1). The salt content in some SDAP sources can also be relatively high and could be a limiting factor for the use of high levels of inclusion. Salt concentration can vary depending on the concentration procedure used before drying and on the anticoagulant used. Thus SDAP sources concentrated by reverse osmosis or vacuum drying may have a higher salt content than SDAP concentrated by ultra filtration (10.9 vs. 4.1\%). Similarly, the use of tri-sodium citrate as anticoagulant also results in a higher salt content than the use of tri-sodium phosphate. It can be concluded that spray dried plasma can be used in piglet diets up to $10 \%$ of inclusion, as long as a correct nutrient balance is maintained.

The response to plasma according to the age of the piglets at weaning was also studied (Table 5). Three age groups were considered 10-17, 18-24 and 25-32 days. Rooke et al. (2003) have shown that the concentrations in blood of specific colostral antibodies transferred from the sow to the piglets decline quadratically during the first weeks of life. They observed a rapid reduction in their concentration between 2 and 14 days of age, and a much slower decline thereafter until day 34 . These authors also described that the total IgG concentration did not change between days 7 and 28 of age, but increased between days 28 and 35 suggesting an activation of the pig's own immune system at around four weeks of age. Therefore for the three age groups considered it could be assumed that: i) piglets of 10 to 17 days of age still have some degree of protection from the colostral antibodies, but their own immune system is immature; ii) that piglets of 18 to 24 days of age have a substantially reduced protection from colostral antibodies and an immature immune system; and iii) that the piglets of 25 to 32 days of age also have a reduced protection from colostral antibodies, but their own immune system is at the initial stages of development. No statistically significant differences between weaning age groups on the effect of SDAP on weight gain or feed efficiency were observed. However, a significantly higher feed intake for the 18-24d age group was observed during the first week post-weaning, and numerically, the response in weight gain and feed efficiency for this age group was also higher. The higher response to SDAP for this age group, coincides with their supposed poorest immune protection, and agrees with a possible support of SDAP to the immune system of the piglets via its IgG content or other mechanisms. In a trial by Torrallardona et al. (2002) although no interaction between weaning age and SDAP could be observed in piglets weaned at 22 or 32 days, a higher response for the younger animals is suggested, which supports the above observations. The importance of SDAP in the very young piglet is also supported by the observation that the inclusion of SDPP in creep feed improves the subsequent postweaning performance (Van Dijk et al., 2001b). 
Table 6. Effect of an experimental health-challenge to piglets on their performance response to spray dried plasma in the first one or two weeks after weaning

\begin{tabular}{|c|c|c|c|c|c|c|c|c|}
\hline \multirow{2}{*}{ Challenged } & \multicolumn{4}{|c|}{ One week post-weaning } & \multicolumn{4}{|c|}{ Two weeks post-weaning } \\
\hline & $\mathrm{n}$ & $\Delta \mathrm{ADG}(\mathrm{g} / \mathrm{d})$ & $\Delta$ ADFI (g/d) & $\Delta$ FGR (g/g) & $\mathrm{n}$ & $\Delta \mathrm{ADG}(\mathrm{g} / \mathrm{d})$ & $\Delta \mathrm{ADFI}(\mathrm{g} / \mathrm{d})$ & $\Delta$ FGR $(\mathrm{g} / \mathrm{g})$ \\
\hline$\overline{\text { YES }}$ & 10 & $+38^{*}$ & $+21^{\mathrm{b},} \dagger$ & $-0.95^{\mathrm{a}, *}$ & 23 & $+46^{*}$ & $+37 *$ & $-0.39^{\mathrm{a}, *}$ \\
\hline NO & 74 & $+54^{*}$ & $+47^{\mathrm{a}, *}$ & $-0.11^{\mathrm{b}}$ & 120 & $+36^{*}$ & $+41^{*}$ & $-0.04^{b}$ \\
\hline Pooled STD & & 37.7 & 36.5 & 0.68 & & 40.7 & 43.8 & 0.43 \\
\hline
\end{tabular}

$\overline{\mathrm{a}, \mathrm{b}}$ Values in same column with different letters are significantly different as analysed by Tukey’s (HSD) test $(\mathrm{p}<0.05)$. n: Number of trials

${ }^{*} p<0.05 ; \dagger p<0.1$. Statistical significance of improvement over controls without plasma.

\section{SPRAY DRIED ANIMAL PLASMA UNDER INFECTIOUS CHALLENGE AND COMBINATION WITH MEDICATED FEED}

Table 6 shows the effect of spray dried plasma according to whether the piglets had been subjected to an experimental health challenge or not. It appears that plasma improves feed to gain ratio more markedly when the piglets are exposed to an experimental challenge. This could be indicative of a lower expenditure of energy and nutrients to build an immune response against the challenge. Studies in which SDPP has been tested under different health environments conclude that the benefits from plasma are significantly higher under lower health conditions, which supports this view. Coffey and Cromwell (1995) observed that weight gain and feed intake were clearly enhanced by SDPP under a conventional environment, but that the response to SDPP was much smaller when pigs were kept in a very clean environment, which resulted in a significant SDPP by environment interaction. Stahly et al. (1995) also tested the effect of adding $6 \%$ of SDAP in the diet with piglets that had been either reared under a conventional environment (high antigen exposure) or via a medicated early weaning scheme (low antigen exposure). They also observed a significant interaction between SDAP and environment so that SDAP improved weigh gain, feed intake and feed efficiency in high antigen exposed pigs but not in low antigen exposed pigs. Similarly, Bergstrom et al. (1997) also concluded that high health segregated early weaning pigs responded less to SDAP than pigs with a lower health status conventionally reared in an on-site nursery. Bregendahl et al. (1998) also observed that the addition of 5\% SDAP in pre-starter feeds improved weight gain by $34 \%$ under a dirty environment, but only by $10 \%$ under a clean environment. For antibiotics, it has also been observed that they have little or no effect on growth when the animals are kept under clean conditions (Roura et al., 1992), which supports the hypothesis that the modes of action of antibiotics and SDAP may share similar mechanisms.

A more marked improvement in FGR in response to SDAP is also observed if feed does not contain medication (Table 7). It has to be noted, however, that in many of the trials considered in this review, animals submitted to an experimental challenge were also offered feed that was not medicated, and therefore both factors may be confused to some degree. Trials evaluating plasma and presence or absence of medication together have shown no interaction between the two factors, so that both effects are additive (Rojas et al., 1994; Coffey and Cromwell, 1995; Torrallardona et al., 2002; Bosi et al., 2004). Cain and Zimmerman (1997) observed that plasma was effective against rotavirus but not against $E$. coli, which could explain the additivity of plasma and antimicrobials. Other studies however, have shown tendencies or significant interactions between SDAP and antimicrobials (Torrallardona et al., 2003; Bikker et al., 2004). In most studies in which SDAP has been compared directly against antibiotics (Rojas et al., 1994; Coffey and Cromwell, 1995; Torrallardona et al., 2002, 2003, 2007; Bikker et al., 2004; Bosi et al., 2004; Conde, 2005), organic acids (OwusuAsiedu et al., 2003b; Torrallardona et al., 2007), other sources of immunoglobulins (Owusu-Asiedu et al., 2002, 2003a,b), plant extracts (Nofrarias et al., 2006), zinc oxide (Owusu-Asiedu et al., 2003b) or carbadox (Owusu-Asiedu et al., 2003b) SDAP results in superior or equivalent results. Srichana et al. (2004) observed that whereas the effect of SDAP occurs mainly in the first week post-weaning that of antibiotics persists for many weeks. The reduction of SDAP

Table 7. Effect of feed medication on the performance response of piglets to spray dried plasma in the first one or two weeks after weaning

\begin{tabular}{|c|c|c|c|c|c|c|c|c|}
\hline \multirow{2}{*}{ Medication } & \multicolumn{4}{|c|}{ One week post-weaning } & \multicolumn{4}{|c|}{ Two weeks post-weaning } \\
\hline & $\mathrm{n}$ & $\Delta \mathrm{ADG}(\mathrm{g} / \mathrm{d})$ & $\Delta$ ADFI (g/d) & $\Delta$ FGR (g/g) & $\mathrm{n}$ & $\Delta \mathrm{ADG}(\mathrm{g} / \mathrm{d})$ & $\Delta$ ADFI (g/d) & $\Delta$ FGR (g/g) \\
\hline$\overline{\text { YES }}$ & 69 & $+55^{*}$ & $+50^{\mathrm{a}, *}$ & $-0.09^{b}$ & 110 & $+36 *$ & $+43^{*}$ & $-0.02^{\mathrm{b}}$ \\
\hline NO & 15 & $+37 *$ & $+17^{\mathrm{b},} \dagger$ & $-0.72^{\mathrm{a}, *}$ & 33 & $+41 *$ & $+32 *$ & $-0.34^{\mathrm{a}, *}$ \\
\hline Pooled STD & & 37.4 & 35.3 & 0.689 & & 40.8 & 43.5 & 0.428 \\
\hline
\end{tabular}

${ }^{\mathrm{a}, \mathrm{b}}$ Values in same column with different letters are significantly different as analysed by Tukey's (HSD) test (p<0.05). n: Number of trials

$* \mathrm{p}<0.05 ; \dagger \mathrm{p}<0.1$. Statistical significance of improvement over controls without plasma. 

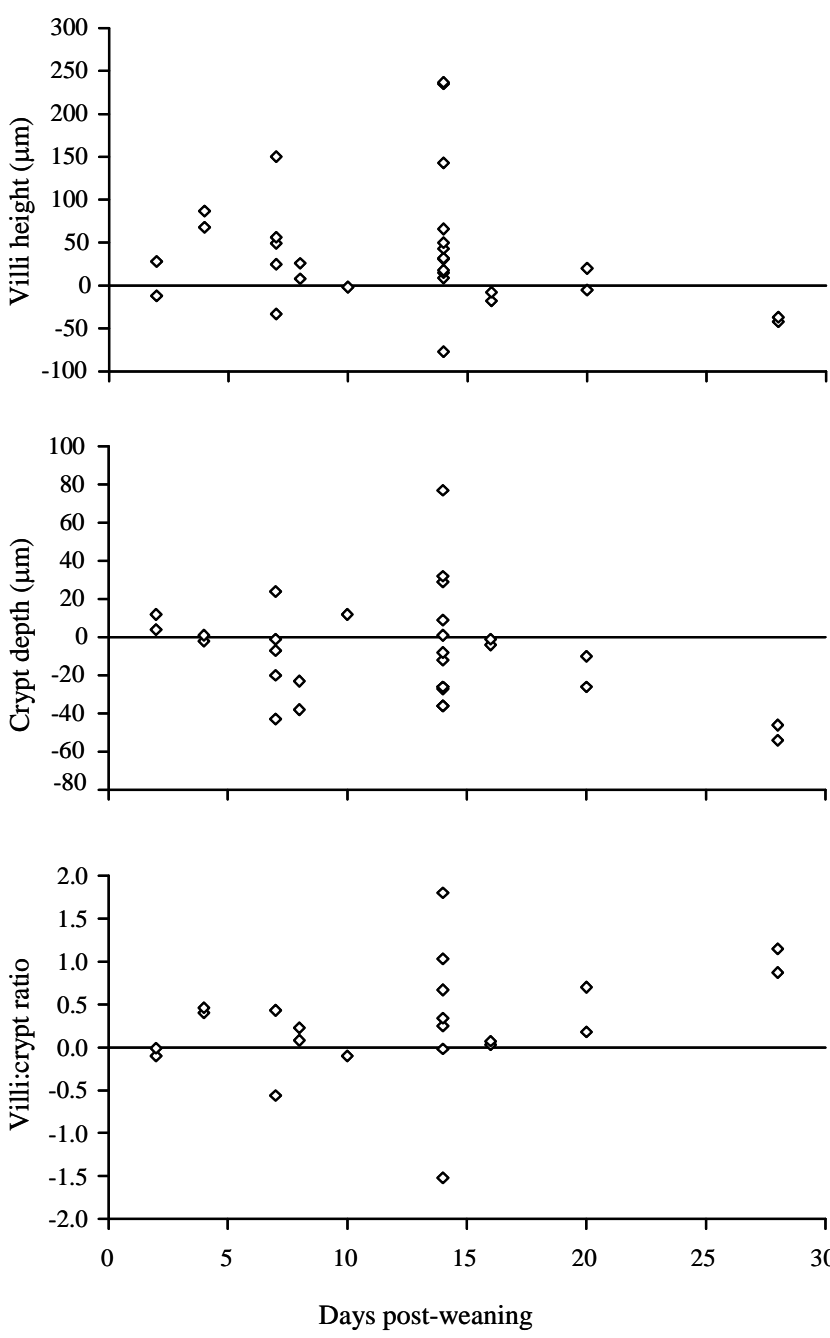

Figure 4. Effect of feeding spray dried plasma to piglets on the change in villus height, crypt depth and villus:crypt ratio relative to the control at different days post-weaning. Number of trials (n) $=30$ for villi height, $\mathrm{n}=30$ for crypt depth and $\mathrm{n}=22$ for $\mathrm{V}: \mathrm{C}$ ratio.

efficacy beyond the third week post-weaning may be explained in part by the reduction in its level of inclusion.

\section{MODE OF ACTION OF SPRAY DRIED ANIMAL PLASMA}

Plasma is a protein source of high interest for pre-starter piglet diets. Most studies evaluating SDAP have shown to improve growth, feed intake and in some cases feed conversion (Table 2). Some authors claim that the beneficial effect of plasma results from the associated increase in feed intake, whereas others maintain that they are the result of the action of its specific bioactive components. Those supporting that the effect is mediated by an increase in the palatability of the diet and the corresponding increase in feed intake base their hypothesis on the observations of
Ermer et al. (1994) for a higher preference for SDAP over dry skim milk feeds. However, Torrallardona and SolàOriol (2009) in a series of double free-choice tests, observed that SDPP preference was intermediate among fourteen common protein sources tested and in some cases SDPP's preference was much lower than that of protein sources (e.g. fishmeal) shown to result in worse productive results. This suggests that the increased feed intake observed for the SDAP containing diets is not a palatability mediated effect. It is possible that the higher feed intake is a consequence of the effect of these diets on the health of the piglets (Torrallardona et al., 2003). It is well known that macrophages secrete cytokines in response to antigenic stimuli, and that cytokines act in the brain to reduce feed intake (Johnson, 1997). Therefore the increased feed intake observed for the SDAP containing diets could be due to a reduction in the pro-inflammatory cytokines produced as a consequence of a lower antigenic load with SDAP containing diets. In pair feeding trials, piglets offered the same amount of feed with or without plasma have shown that the effects of plasma are independent of intake, which suggests a specific biological effect (Jiang et al., 2000b). In weaning pigs, feed intake has been shown to be closely correlated with the integrity of the gut mucosa (Pluske et al., 1997). Figure 4 shows the changes in villi and crypt morphology due to SDAP measured at different days postweaning obtained form different studies (Jiang et al., 2000b; Owusu-Asiedu et al., 2002, 2003a,b; Torrallardona et al., 2003, 2007; Conde, 2005; Nofrarias et al., 2006). SDAP clearly increases villus height (particularly in the first two weeks post-weaning), but its effect on crypt depth is not as clear.

The most widely accepted hypothesis for the mode of action for plasma is probably that its immunoglobulin content is biologically active against pathogens and enterotoxins. This was initially supported by studies in which plasma was proven to reduce the incidence in postweaning diarrhoea (Gatnau and Zimmerman, 1991; Peet Schwering et al., 1995; Cain and Zimmerman, 1997). Furthermore, studies testing SDPP under environments of different health status have shown that the benefits of plasma are significantly higher under poor sanitary conditions (Coffey and Cromwell, 1995; Stahly et al., 1995; Bergstrom et al., 1997; Bregendahl et al., 1998). Gatnau et al. (1995), Owen et al. (1995), Weaver et al. (1995) and Pierce et al. (2005) have separated plasma into high, medium and low molecular weight fractions, representing globulins, albumin and fibrin, respectively and compared their efficacy with that of SDAP. Their results show that the high molecular weight (immunoglobulin) fraction is the responsible for the beneficial effects of plasma. Godfredson-Kisic et al. (1999) showed that a diet with $2 \%$ porcine globulin concentrate, is as effective in maintaining 
growth and intake of recently weaned piglets as a diet with $8 \%$ plasma. Immunoglobulins keep their biological activity after processing the plasma (Gatnau et al., 1989). They can reach the proximal intestine of recently weaned piglets without being digested, most likely preserving their ability to bind to bacteria and virus (Morel, 1995). The destruction of SDAP antibodies by autoclaving has been shown to reduce its efficacy (Owusu-Asiedu et al., 2002), although it cannot be ruled out that other bioactive components in plasma could also be damaged with this procedure.

Plasma contains antibodies against pathogenic bacteria such as enterotoxic E. coli (Owusu-Asiedu et al., 2002). Therefore, plasma immunoglobulins may provide antimicrobial protection, reduce the intestinal immune system activation and prevent mucosal damage by pathogenic bacteria in particularly susceptible animals such as the newly-weaned pig. It has been shown that plasma with guaranteed high levels of immunoglobulins is superior to conventional plasma (Bosi et al., 2001; Conde, 2005). In Bosi et al. (2001)'s study, piglets challenged with $E$. coli K88 and fed plasma with high IgG levels had a lower concentration of specific IgA against K88 in plasma and saliva. This suggests a protective effect against the adhesion E. coli K88, since IgA production against a specific bacteria, requires adhesion of this bacteria (using its fimbriae) to the enterocytes in the mucosal villi. Since IgG cannot be absorbed through the intestinal wall in 3-4 week old piglets, they have to act in the intestinal lumen. IgG bind to the virus or bacteria, avoid their union to the enterocytes and prevent the colonisation and damage of the intestinal wall. This is supported by the observations of Perez-Bosque et al. (2004) in challenged rats, who observed a lower activation of the intestinal immune system with the use of SDAP. It has also been hypothesised that the IgG against soyabean proteins present in SDAP, could help weaning piglets to adapt better to the weaning diet rich in highly antigenic soybean proteins. However this could not be demonstrated in a trial designed for this purpose (Hartke et al., 2003).

Glycoproteins have been proposed for being responsible for SDAP's effects instead of (or in addition to) immunoglobulins, by impeding antigen binding. Thus Sanchez et al. (1993) showed in vitro that certain glycoproteins obtained from plasma could act as a binding site for E. coli fimbriae, and Mouricout and Julien (1986) observed that bovine plasma glycoproteins inhibited $E$. coli adhesion to enterocytes. Subsequently in a study in calves, Mouricout et al. (1990), saw that glycoproteins obtained from bovine plasma inhibited intestinal adhesion of $E$. coli and protected the calves which had been deprived of colostrum against lethal doses of the bacteria. Nollet et al. (1999), in studies of piglets, used a plasma source without specific immunoglobulins for $E$. coli F18, and showed that it impeded E. coli F18 binding to enterocytes by receptor competition, which suggested a non-specific protection mechanism. Challenge studies in which diarrhoea was evaluated have shown that SDAP improves faecal scores (Owusu-Asiedu et al., 2003a,b; Conde, 2005), and body condition (Van Dijk et al., 2002a). Torrallardona et al. (2003) observed increased Lactobacilli counts in ileum and caecum of piglets fed SDAP, suggesting that SDAP promotes a beneficial microbiota. This observation however, could not be confirmed in posterior studies by the same group (Conde, 2005; Torrallardona et al., 2007). In experiments with piglets, Jiang et al. (2000b) saw that plasma increased efficiency in dietary protein utilisation and reduced intestinal mass, cell density between villi in the lamina propia and circulatory urea concentration, which the authors attributed to a minimisation in amino acid catabolism by the microflora and an increase in dietary amino acid availability for growth. According to Jiang et al. (2000a), SDAP consumption reduces dietary amino acid catabolism in the intestine, and increases the efficiency of dietary protein utilisation, enabling greater availability of dietary amino acids for growth of lean tissue.

Touchette et al. (2002) showed a lower basal activation of the immune system in piglets fed SDAP than in piglets without SDAP. The same group of researchers described an increased activation of the hypothalamic-pituitary-adrenal (HPA) axis in SDAP-fed piglets following a challenge with LPS or E. coli (Touchette et al., 1999; Carroll et al., 2002). Carroll et al. (2002) proposed two mechanisms by which plasma prevents HPA axis activation. The first is a direct effect of plasma, preventing growth and colonisation of antigenic bacteria in the small intestine, by immunoglobulins. The second is an indirect effect of plasma on mucosal integrity, promoting intestinal growth, improving villi height and the villus height: crypt depth ratio as shown in previous studies (Spencer et al., 1997; Touchette et al., 1997). This would enable the animals to have a better barrier, which would prevent potential pathogens from crossing the intestinal wall. Both mechanisms would reduce HPA axis activation in piglets, reducing immunological stress, and immune system stimulation. Immune system activation has been shown to be associated with reduced intake and growth (van Heugten et al., 1994), due to the increase in pro-inflammatory cytokines which inhibit growth and intake (Johnson, 1997), and with a change in nutrient distribution, no longer used for skeletal muscle growth but for supporting the energy expenditure necessary for the immune system (Klaising and Johnstone, 1991). Bosi et al. (2004) confirmed a reduction in the production of the pro-inflammatory cytokines (tumour necrosis factor- $\alpha$, interleukin-8 and interferon- $\gamma$ ) in the jejunum of SDAP-fed piglets challenged with enterotoxigenic E. coli (ETEC) K88. Therefore the reduction in immune system activation, which results from 
the consumption of diets with SDAP by piglets, would improve their intake and growth (Touchette et al., 2002).

\section{CONCLUDING REMARKS}

Plasma is a widely accepted ingredient for pre-starter piglet diets that has been shown to improve growth, feed intake and/or feed conversion. There is evidence suggesting that the improvement in feed intake is due to a reduction in the production of pro-inflammatory cytokines as a consequence of a lower antigenic load when SDAP is provided, rather than to an improvement in feed palatability. The superiority of SDAP over all the other protein sources it has been compared to (even those that have been shown to have equivalent or better nutritional value) is against a nutritionally driven effect. Most of the evidence provided suggests a specific action of the IgG or other bioactive substances in SDAP against porcine pathogens. IgG and other bioactive substances may prevent the binding of pathogens to the gut wall, reduce the activation of the immune system and reduce the incidence of diarrhoea in the post-weaning phase. The specificity of the IgG could explain the better efficacy of plasma from porcine origin over that of plasma from bovine or mixed origin. The suggested higher efficacy of SDAP for piglets weaned at 18-24 days of age (coinciding with a low protection from colostral antibodies and an immature immune system of the piglet), also supports an IgG mediated mode of action. Finally, as for antibiotics, SDAP efficacy is much higher under poor sanitary conditions (i.e. experimental challenge or absence of medication in the feed). In conclusion spray dried plasma appears to prevent the adhesion of pathogenic bacteria to the gut wall, reduces de activation of the immune system and the production of pro-inflammatory cytokines (thus avoiding a reduction in feed intake), and reduces the needs for energy and nutrients to build an immune response against the challenge, which results in better growth. For all this plasma can be postulated as an excellent alternative to in-feed antimicrobials for piglets in the post-weaning phase.

\section{REFERENCES}

Angulo, E. and D. Cubiló. 1998. Effect of different dietary concentrations of spray-dried porcine plasma and a modified soyprotein product on the growth performance of piglets weaned at $6 \mathrm{~kg}$ body weight. Anim. Feed Sci. Technol. 72:7179.

Bergstrom, J. R., J. L. Nelssen, M. D. Tokach, R. D. Goodband, S. S. Dritz, K. Q. Owen and W. B. Nessmith, Jr. 1997. Evaluation of spray-dried animal plasma and select menhaden fish meal in transition diets of pigs weaned at 12 to 14 days of age and reared in different production systems. J. Anim. Sci. 75:30043009.
Bikker, P., A. J. van Dijk, A. Dirkzwager, J. Fledderus, M. UbbinkBlanksma and A. C. Beynen. 2004. The influence of diet composition and an anti-microbial growth promoter on the growth response of weaned piglets to spray dried animal plasma. Livest. Prod. Sci. 86:201-208.

Borg, B. S., J. M. Campbell, L. E. Russel, C. Rodríguez and J. Ródenas. 2002. Evaluation of the chemical and biological characteristics of spray-dried plasma protein collected from various locations around the world. Proc. Am. Assoc. Swine Vet. 33:97-100.

Bosi, P., I. K. Han, H. J. Jung, K. N. Heo, S. Perini, A. M. Castellazzi, L. Casini, D. Creston and C. Gremokolini. 2001. Effect of different spray dried plasmas on growth, ileal digestibility, nutrient deposition, immunity and health of earlyweaned pigs challenged with E. coli k88. Asian-Aust. J. Anim. Sci. 14:1138-1143.

Bosi, P., L. Casini, A. Finamore, C. Cremokolini, G. Merialdi, P. Trevisi, F. Nobili and E. Mengheri. 2004. Spray-dried plasma improves growth performance and reduces inflammatory status of weaned pigs challenged with enterotoxigenic Escherichia coli K88. J. Anim. Sci. 82:1764-1772.

Bregendahl, K., J. C. Sparks, J. Bassaganya and D. R. Zimmerman. 1998. Effects of spray-dried plasma and a product of hydrolyzed porcine intestines on weanling pigs raised in two environments with different health-status. J. Anim. Sci. 76(Suppl. 1):181(Abstr.).

Bruininx, E., C. M. C. van der Peet-Schwering, J. W. Schrama, L. A. den Hartog, H. Everts and A. C. Beynen. 2001. The IVOG $^{\circledR}$ feeding station: A tool for monitoring the individual feed intake of group-housed weanling pigs. J. Anim. Physiol. Anim. Nutr. 85:81-87.

Burnham, L. L., J. D. Hancock, I. H. Kim, M. R. Cabrera, K. L. Larsen and R. H. Hines. 1995. Spray-dried wheat gluten and porcine plasma protein for nursery pigs. J. Anim. Sci. 73(Suppl. 1): 171.

Cain, C. M. and D. R. Zimmerman. 1997. Effect of spray dried plasma (SDP) on fecal shedding of hemolytic escherichia coli (HEC) and rotavirus by pigs in a segregated early-weaned (SEW) enviroment. J. Anim. Sci. 75(Suppl. 1):61(Abstr.).

Campbell, J. M., E. M. Weaver, L. E. Russell, F. Chi and J. D. Arthington. 1998. Evaluation of dietary inclusion of spraydried plasma proteins (AP-920 and Appetein) on pig performance throughout the nursery. J. Anim. Sci. 76(Suppl. 1): 180 .

Carroll, J. A., K. J. Touchette, R. L. Matteri, C. J. Dyer and G. L. Allee. 2002. Effect of spray-dried plasma and lipopolysaccharide exposure on weaned rigs: II. Effects on the hypothalamic-pituitary-adrenal axis of weaned pigs. J. Anim. Sci. 80:502-509.

Chae, B. J., I. K. Han, J. H. Kim, C. J. Yang, J. D. Hancock, I. H. Kim and D. A. Anderson. 1999. Effects of dietary protein sources on ileal digestibility and growth performance for earlyweaned pigs. Livest. Prod. Sci. 58:45-54.

Coffey, R. D. and G. L. Cromwell. 1995. The impact of environment and antimicrobial agents on the growth response of early-weaned pigs to spray-dried porcine plasma. J. Anim. Sci. 73:2532-2539.

Coffey, M. T. and G. L. Cromwell. 2001. Use of spray-dried animal plasma in diets for weanling pigs. Pigs News and 
Information 22:39N-48N.

Conde, R. 2005. SDAP in piglet nutrition. A possible alternative to antibiotics. Ph.D. Thesis, Autonomous University of Barcelona, Bellaterra, Spain.

DANMAP. 2004. Use of antimicrobial agents and occurrence of antimicrobial resistance in bacteria from food animals, foods and humans in denmark, Danish Institute for Food and Veterinary Research.

de Rodas, B. Z., K. S. Sohn, C. V. Maxwell and L. J. Spicer. 1995. Plasma protein for pigs weaned at 19 to 24 days of age: Effects on performance and plasma insulin-like growth factor I, growth hormone, insulin and glucose concentrations. J. Anim. Sci. 73:3657-3665.

DeRouchey, J. M., M. D. Tokach, J. L. Nelssen, R. D. Goodband, S. S. Dritz, J. C. Woodworth, B. W. James, M. J. Webster and C. W. Hastad. 2004. Evaluation of methods to reduce bacteria concentrations in spray-dried animal plasma and its effects on nursery pig performance. J. Anim. Sci. 82:250-261.

Easter, R. A. 1988. Acidification of diets for pigs. In: Recent advances in animal nutrition (Ed. W. Haresing and D. J. A. Cole). Butterworths, London. pp. 61-71.

Ermer, P. M., P. S. Miller and A. J. Lewis. 1994. Diet preference and meal patterns of weanling pigs offered diets containing either spray-dried porcine plasma or dried skim milk. J. Anim. Sci. 72:1548-1554.

Gatnau, R., P. S. Paul and D. R. Zimmerman. 1989. Spray dried porcine plasma as a source of immunoglobulins for newborn piglets. J. Anim. Sci. 67(Suppl. 1):244.

Gatnau, R. and D. R. Zimmerman. 1990. Spray dried porcine plasma (SDPP) as a source of protein for weanling pigs. J. Anim. Sci. 68(Suppl. 1):374(Abstr.).

Gatnau, R. and D. R. Zimmerman. 1991. Spray dried porcine plasma (SDPP) as a source of protein for weanling pigs in two environments. J. Anim. Sci. 69(Suppl. 1):103(Abstr.).

Gatnau, R., D. R. Zimmerman, T. Diaz and J. Johns. 1991. Determination of optimum levels of spray dried porcine plasma (SDPP) in diets for weanling pigs. J. Anim. Sci. 69(Suppl. 1):369(Abstr.).

Gatnau, R. and D. R. Zimmerman. 1992. Determination of optimum levels of inclusion of spray dried porcine plasma (SDPP) in diets for weanling pigs fed in practical conditions. J. Anim. Sci. 70(Suppl. 1):60(Abstr.).

Gatnau, R. and D. R. Zimmerman. 1994. Effects of spray-dried plasma of different sources and processes on growth performance of weanling pigs. J. Anim. Sci. 72(Suppl. 1):166(Abstr.).

Gatnau, R., C. Cain, M. Drew and D. R. Zimmerman. 1995. Mode of action of spray-dried porcine plasma in weanling pigs. J. Anim. Sci. 73(Suppl. 1):82(Abstr.).

Godfredson-Kisic, J. A., T. E. Shipp and K. C. Ferrell. 1999. The effects of various concentrated immunoglobulin protein sources in phase 1 diets of early weaned pigs. J. Anim. Sci. 77(Suppl. 1):174(Abstr.).

Grinstead, G. S., R. D. Goodband, S. S. Dritz, M. D. Tokach, J. L. Nelssen, J. C. Woodworth and M. Molitor. 2000. Effects of a whey protein product and spray-dried animal plasma on growth performance of weanling pigs. J. Anim. Sci. 78:647657.

Hampson, D. J. 1986. Alterations in piglet small intestinal structure at weaning. Res. Vet. Sci. 40:32-40.

Hansen, J. A., J. L. Nelssen, R. D. Goodband and T. L. Weeden. 1993. Evaluation of animal protein supplements in diets of early-weaned pigs. J. Anim. Sci. 71:1853-1862.

Hartke, J. L., G. A. Apgar, K. E. Griswold, B. N. Jacobson, T. L. Rosenthal and T. A. Guthrie. 2003. Responses of weanling pigs to spray-dried animal plasma added to simple diets containing varying levels of soya-bean meal. Anim. Sci. 77:73-78.

Hunziker, W. and J. P. Kraehenbuhl. 1998. Epithelial transcytosis of immunoglobulins. J. Mammary Gland Biology and Neoplasia 3:287-302.

Husband, A. J. and M. A. Bennell. 1980. Intestinal immunity in pigs. Pigs News and Information 1:211-213.

Jensen, M. S., S. K. Jensen and K. Jakobsen. 1997. Development of digestive enzymes in pigs with emphasis on lipolytic activity in the stomach and pancreas. J. Anim. Sci. 75:437-445.

Jiang, R. H., X. Y. Chang, B. Stoll, K. J. Ellis, R. J. Shypailo, E. Weaver, J. Campbell and D. G. Burrin. 2000a. Dietary plasma protein is used more efficiently than extruded soy protein for lean tissue growth in early-weaned pigs. J. Nutr. 130:20162019.

Jiang, R. H., X. Y. Chang, B. Stoll, M. Z. Fan, J. Arthington, E. Weaver, J. Campbell and D. G. Burrin. 2000b. Dietary plasma protein reduces small intestinal growth and lamina propria cell density in early weaned pigs. J. Nutr. 130:21-26.

Johnson, R. W. 1997. Inhibition of growth by pro-inflammatory cytokines: An integrated view. J. Anim. Sci. 75:1244-1255.

Kats, L. J., M. D. Tokach, R. D. Goodband and J. L. Nelssen. 1992. Influence of protein source fed to the early weaned pigs during phase I (d 0-9) on the response to various protein sources fed during phase II (d 9-28). J. Anim. Sci. 70(Suppl. 1):60(Abstr.).

Kats, L. J., J. L. Nelssen, M. D. Tokach, R. D. Goodband, J. A. Hansen and J. L. Laurin. 1994. The effect of spray-dried porcine plasma on growth-performance in the early-weaned pig. J. Anim. Sci. 72:2075-2081.

Kelly, D., J. A. Smyth and K. J. McCraken. 1991. Digestive development of the early-weaned pig 1 . Effect of continuous nutrient supply on the development of the digestive tract and on changes in digestive enzyme activity during the first week post-weaning. Br. J. Nutr. 65:169-180.

Kerr, C. A., R. D. Goodband, J. W. Smith, II, R. E. Musser, J. R. Bergstrom, W. B. Nessmith, Jr., M. D. Tokach and J. L. Nelssen. 1998. Evaluation of potato proteins on the growth performance of early-weaned pigs. J. Anim. Sci. 76:3024-3033.

Kidder, D. E. and M. J. Manners. 1980. The level and distribution of carbohydrases in the small intestine mucosa of pigs from 3 weeks of age to maturity. Br. J. Nutr. 43:141-153.

Kim, S. W. and R. A. Easter. 2001. Nutritional value of fish meals in the diet for young pigs. J. Anim. Sci. 79:1829-1839.

Klaising, K. C. and B. J. Johnstone. 1991. Monokines in growth and development. Poult. Sci. 70:1781-1789.

Lawrence, K. R., R. D. Goodband, M. D. Tokach, S. S. Dritz, J. L. Nelssen and J. M. DeRouchey. 2004. Comparison of wheat gluten and spray-dried animal plasma in diets for nursery pigs. J. Anim. Sci. 82:3635-3645.

Makkink, C. A., G. P. Negulescu, Q. Guixin and M. W. A. Verstegen. 1994. Effect of dietary protein souce on feed intake, growth, pancreatic enzyme activities and jejunal morphology in newly-weaned piglets. Br. J. Nutr. 72:353-368. 
Miller, B. G., P. S. James, M. W. Smith and F. J. Bourne. 1986. Effect of weaning on the capacity of pig intestinal villi to digest and absorb nutrients. J. Agric. Sci. 107:579-589.

Monks, J. and M. C. Neville. 2004. Albumin transcytosis across the epithelium of the lactating mouse mammary gland. J. Physiol. (Lond.) 560:267-280.

Morel, P. C. H., L. M. Schollum, T. R. Buwalda and G. Pearson. 1995. Digestibility of bovine immunoglobulin in the piglet. In: Proceedings of the bienal APSA conferences. Manipulating pig production V. p 181.

Mouricout, M. and R. A. Julien. 1986. Inhibition of mannoseresistant haemagglutination of sheep erythrocytes by enterotoxigenic escherichia coli in the presence of plasma glycoprotein glycans. FEM Microbiology Letters 37:145-149.

Mouricout, M., J. M. Petit, J. R. Carias and R. Julien. 1990. Glycoprotein glycans that inhibit adhesion of E. coli mediated by K99 fimbriae: Treatment of experimental colibacillosis. Infec. Immun. 58:98-106.

National Research Council 1998. Nutrient Requirements of Swine. 10th Ed. National Academy Press, Washington, DC.

Nessmith, Jr, W. B., M. D. Tokach, R. D. Goodband, J. L. Nelssen, J. R. Bergström, J. W. S. II, K. Q. Owen and B. T. Richert. 1995. The effects of substituting spray-dried whole egg from grading plants only for spray-dried animal plasma in phase I diets. J. Anim. Sci. 73(Suppl. 1):171(Abstr.).

Nessmith, W. B., Jr., J. L. Nelssen, M. D. Tokach, R. D. Goodband, J. R. Bergstrom, S. S. Dritz and B. T. Richert. 1997. Evaluation of the interrelationships among lactose and protein sources in diets for segregated early-weaned pigs. J. Anim. Sci. 75:32143221.

Niewold, T. A., A. J. van Dijk, P. L. Geenen, H. Roodink, R. Margry, and J. van Der Meulen. 2007. Dietary specific antibodies in spray-dried immune plasma prevent enterotoxigenic Escherichia coli F4 (ETEC) post-weaning diarrhoea in piglets. Vet. Microbiol. 124:362-369.

Nofrarias, M., E. G. Manzanilla, J. Pujols, X. Gibert, N. Majó, J. Segalés and J. Gasa. 2006. Effects of spray-dried porcine plasma and plant extracts on intestinal morphology and on leukocyte cell subsets of weaned pigs. J. Anim. Sci. 84:27352742.

Nollet, H., P. Deprez, E. Van Driessche and E. Muylle. 1999. Protection of just weaned pigs against infection with f18(+) Escherichia coli by non-immune plasma powder. Vet. Microbiol. 65:37-45.

Owen, K. Q., J. L. Nelssen, R. D. Goodband, M. D. Tokach, K. G. Friesen, B. T. Richert, J. W. Smith and L. E. Russell. 1995. Effect of various fractions of spray-dried porcine plasma on performance of early weaned pigs. J. Anim. Sci. 73(Suppl. 1):81(Abstr.).

Owusu-Asiedu, A., S. K. Baidoo, C. M. Nyachoti and R. R. Marquardt. 2002. Response of early-weaned pigs to spraydried porcine or animal plasma-based diets supplemented with egg-yolk antibodies against enterotoxigenic Escherichia coli. J. Anim. Sci. 80:2895-2903.

Owusu-Asiedu, A., C. M. Nyachoti, S. K. Baidoo, R. R. Marquardt and X. Yang. 2003a. Response of early-weaned pigs to an enterotoxigenic Escherichia coli (K88) challenge when fed diets containing spray-dried porcine plasma or pea protein isolate plus egg yolk antibody. J. Anim. Sci. 81:1781-1789.

Owusu-Asiedu, A., C. M. Nyachoti and R. R. Marquardt. 2003b. Response of early-weaned pigs to an enterotoxigenic Escherichia coli (K88) challenge when fed diets containing spray-dried porcine plasma or pea protein isolate plus egg yolk antibody, zinc oxide, fumaric acid, or antibiotic. J. Anim. Sci. 81:1790-1798.

Peet Schwering, C. M. C. V. D., G. P. Binnendijk, and C. M. C. Van der Peet Schwering. 1995. The effect of spray-dried porcine plasma in diets with different protein sources on the performance of weanling piglets. Proefverslag Proefstation voor de Varkenshouderij No. 4.127:24 pp.

Peet Schwering, C. M. C. V. D., G. P. Binnendijk and C. M. C. Van der Peet Schwering. 1997. Spray-dried blood plasma and spray-dried blood cells in diets of weaned piglets. Proefverslag Proefstation voor de Varkenshouderij No. 1.168:1-20.

Pérez-Bosque, A., C. Pelegrí, M. Vicario, M. Castell, L. Russell, J. M. Campbell, J. D. Quigley, J. Polo, C. Amat and M. Moretó. 2004. Dietary plasma protein affects the immune response of weaned rats challenged with S. aureus superantigen B. J. Nutr. 134:2667-2672.

Pierce, J. L., G. L. Cromwell, M. D. Lindemann, L. E. Russell and E. M. Weaver. 2005. Effects of spray-dried animal plasma and immunoglobulins on performance of early weaned pigs. J. Anim. Sci. 83:2876-2885.

Pluske, J. R., I. H. Williams and F. X. Aherne. 1991. Maintenance of villous height and crypt depth in the small intestine of weaned pigs. In: Manipulating Pig Production III. Australasian pig Science Association, Werribbee, Australia. p. 143.

Pluske, J. R., D. J. Hampson and I. H. Williams. 1997. Factors influencing the structure and function of the small intestine in the weaned pig: A review. Livest. Prod. Sci. 51:215-236.

Polo, J., J. D. Quigley, L. E. Russell, J. M. Campbell, J. Pujols and P. D. Lukert. 2005. Efficacy of spray-drying to reduce infectivity of pseudorabies and porcine reproductive and respiratory syndrome (PRRS) viruses and seroconversion in pigs fed diets containing spray-dried animal plasma. J. Anim. Sci. 83:1933-1938.

Rantanen, M. M., J. W. Smith II, B. T. Richert, K. G. Friesen, J. L. Nelssen, R. D. Goodband, M. D. Tokach and L. E. Russell. 1994. Influence of spray dried plasma source on growth performance of weanling pigs. J. Anim. Sci. 72(Suppl. 1):166(Abstr.).

Rojas, J. L., M. H. Jurgens and D. R. Zimmerman. 1994. Effect of spray-dried porcine plasma, antimicrobial agents or their combination on performance of weanling pigs. Swine Reseach Report AS-629, Iowa State University, Ames, IA.

Rooke, J. A., C. Carranca, I. M. Bland, A. G. Sinclair, M. Ewen, V. C. Bland and S. A. Edwards. 2003. Relationships between passive absorption of immunoglobulin $\mathrm{G}$ by the piglet and development of active immunity. Livest. Prod. Sci. 81:223-234.

Roura, E., J. Homedes and K. C. Klasing. 1992. Prevention of immunologic stress contributes to the growth-permitting ability of dietary antibiotics in chicks. J. Nutr. 122:2383-2390.

Russell, L. E. 1994. Effect of plasma source and processing method on postweaning performance of pigs. J. Anim. Sci. 77(Suppl. 1):166 (Abstr.).

Sanchez, R., L. Kanarek, J. Koninkx, H. Hendriks, P. Lintermans, 
A. Bertels, G. Charlier and E. Vandriessche. 1993. Inhibition of adhesion of enterotoxigenic Escherichia-coli-cells expressing F-17 fimbriae to small-intestinal mucus and brush-border membranes of young calves. Microbial Pathogenesis 15:407419.

Smith II, J. W., B. T. Richert, W. B. Nessmith Jr, J. L. Nelssen, R. D. Goodband and M. D. Tokach. 1995. The effect of spraydried plasma source on starter pig performance. J. Anim. Sci. 78(Suppl. 1):80(Abstr.).

Sohn, K. S., C. V. Maxwell and D. S. Buchanan. 1991. Plasma protein as an alternative protein source for early weaned pigs. J. Anim. Sci. 69(Suppl. 1):362(Abstr.).

Spencer, J. D., K. J. Touchette, H. Liu, G. L. Allee, M. D. Newcomb, M. S. Kerley and L. W. Pace. 1997. Effect of spraydried plasma and fructooligosacharide on nursery performance and small intestinal morphology of weaned pigs. J. Anim. Sci. 75(Suppl. 1):199(Abstr.).

Srichana, P., A. M. Gaines, B. W. Ratliff, G. L. Allee, J. D. Crenshaw, J. M. Campbell, J. D. Quigley and L. E. Russell. 2004. Impact of spray-dried plasma with or without antimicrobials on nursery pig performance. J. Anim. Sci. 82(Suppl. 1):140(Abstr.).

Stahly, T. S., D. R. Cook, S. G. Swenson, N. H. Williams and D. R. Zimmerman. 1995. Growth responses of pigs to dietary plasma protein (pp) additions as influenced by pig antigen exposure and pp source. J. Anim. Sci. 73(Suppl. 1):81(Abstr.).

Svendsen, J. and P. Brown. 1973. IgA immunoglobulin levels in porcine sera and mammary secretions. Res. Vet. Sci. 15:65-69.

Svendsen, J. and J. L. Larsen. 1977. Studies of the pathogenesis of enteric $E$. coli infections in weaned pigs. The significance of the milk of the dam in preventing the disease. Nord. Vet. Med. 29:533-538.

Torrallardona, D. and D. Solà-Oriol. 2009. Evaluation of freechoice feedstuffs preference by pigs. In: Voluntary feed intake in pigs (Ed: D. Torrallardona and E. Roura). Wageningen Academic Publishers, Wageningen, The Netherlands. pp. 215242.

Torrallardona, D., R. Conde, E. Esteve-Garcia and J. Brufau. 2002. Use of spray dried animal plasma as an alternative to antimicrobial medication in weanling pigs. Anim. Feed Sci. Technol. 99:119-129.

Torrallardona, D., M. R. Conde, I. Badiola, J. Polo and J. Brufau. 2003. Effect of fishmeal replacement with spray-dried animal plasma and colistin on intestinal structure, intestinal microbiology, and performance of weanling pigs challenged with Escherichia coli K99. J. Anim. Sci. 81:1220-1226.

Torrallardona, D., R. Conde, J. I. Badiola and J. Polo. 2007. Evaluation of spray dried animal plasma and calcium formate as alternatives to colistin in piglets experimentally infected with Escherichia coli K99. Livest. Sci. 108:303-306.

Touchette, K. J., G. L. Allee, M. D. Newcomb, L. W. Pace and M. R. Ellersieck. 1997. Impact of feed intake and spray-dried plasma on nursery performance and intestinal morphology of weaned pigs. J. Anim. Sci. 75(Suppl. 1):199(Abstr.).
Touchette, K. J., G. L. Allee, R. L. Matteri, C. J. Dyer, L. W. Pace, and J. A. Carroll. 1999. Effect of spray-dried plasma and Escherichia coli on intestinal morphology and the hypotathalamic-pituitary-adrenal (HPA) axis of the weaned pig. J. Anim. Sci. 77(Suppl. 1):56(Abstr.).

Touchette, K. J., J. A. Carroll, G. L. Allee, R. L. Matteri, C. J. Dyer, L. A. Beausang and M. E. Zannelli. 2002. Effect of spray-dried plasma and lipopolysaccharide exposure on weaned pigs: I. Effects on the immune axis of weaned pigs. J. Anim. Sci. 80:494-501.

Tumbleson, M. E., D. A. Schmidt and E. Scholl. 1986. Hematology and clinical chemistry. Diseases of swine. Iowa State University Press, Ames, IA, USA.

van Beers-Schreurs, H. M. G., M. J. A. Nabuurs, L. Vellenga, H. J. Kalsbeek-van der Valk, T. Wensing and J. H. Breukink. 1998. Weaning and weanling diet influence the villous height and crypt depth in small intestine of pigs and alter the concentration of short-chain fatty acids in the large intestine and blood. J. Nutr. 128:947-953.

van Dijk, A. J., H. Everts, M. J. A. Nabuurs, R. Margry and A. C. Beynen. 2001a. Growth performance of weanling pigs fed spray-dried animal plasma: A review. Livest. Prod. Sci. 68:263-274.

Van Dijk, A. J., M. Ubbink-Blanksma, J. G. P. Van der Palen and A. C. Beynen. 2001b. Pre- and postweaning performance of piglets fed pre-weaning diets containing either spray-dried porcine plasma, whey protein concentrate or whey powder. J. Anim. Sci. 79(Suppl. 1):22(Abstr.).

Van Dijk, A. J., P. M. M. Enthoven, S. G. C. Van den Hoven, M. Van Laarhoven, T. A. Niewold, M. J. A. Nabuurs and A. C. Beynen. 2002a. The effect of dietary spray-dried porcine plasma on clinical response in weaned piglets challenged with a pathogenic Escherichia coli. Vet. Microbiol. 84:207-218.

Van Dijk, A. J., R. Margry, A. G. Van der Lee, G. Hemke and A. C. Beynen. 2002b. Growth performance and health status in weanling piglets fed spray-dried porcine plasma under typical northern European conditions. J. Anim. Physiol. Anim. Nutr. $86: 17-25$

van Heugten, E., J. W. Spears and M. T. Coffey. 1994. The effect of dietary protein on performance and immune response in weanling pigs subjected to an inflammatory challenge. J. Anim. Sci. 72:2661-2669.

Weaver, E. M., L. E. Russell and M. D. Drew. 1995. The effecr of spray dried animal plasma fractions on performance of newly weaned pigs. J. Anim. Sci. 73(Suppl. 1):81(Abstr.).

Woodgate, S. L., G. Hoyos and K. S. Jackes. 1997. Comparison of protein ingredients in diets for weanling pigs. J. Anim. Sci. 75(Suppl. 1):59(Abstr.).

Yun, J. H., J. S. Yong and B. J. Chae. 2005. Effects of feeding rice protein concentrate on growth performance and ileal digestibility in early-weaned pigs. Asian-Aust. J. Anim. Sci. 18:384-389.

Zimmerman, D. R. 1987. Porcine plasma proteins in diets of weanling pigs. Swine Research Reports, Iowa State University. 